Published in final edited form as:

Science. 2020 January 17; 367(6475): . doi:10.1126/science.aaz5357.

\title{
Correlative three-dimensional super-resolution and block face electron microscopy of whole vitreously frozen cells
}

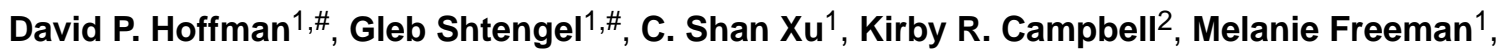 \\ Lei Wang ${ }^{3,4,5, \S}$, Daniel E. Milkie ${ }^{1}$, H. Amalia Pasollii ${ }^{1, \neq}$, Nirmala lyer ${ }^{1}$, John A. Bogovic ${ }^{1}$, \\ Daniel R. Stabley ${ }^{6}$, Abbas Shirinifard ${ }^{7}$, Song Pang ${ }^{1}$, David Peale ${ }^{1}$, Kathy Schaefer ${ }^{1}$, Wim \\ Pomp $^{3,4,5, \dagger}$, Chi-Lun Chang ${ }^{1}$, Jennifer Lippincott-Schwartz ${ }^{1}$, Tom Kirchhausen ${ }^{1,3,4,5}$, David

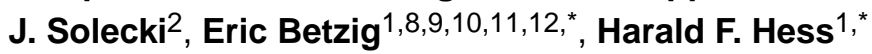 \\ 1Janelia Research Campus, Howard Hughes Medical Institute, Ashburn, VA 20147, USA. \\ 2Department of Developmental Neurobiology, St. Jude Children's Research Hospital, 262 Danny \\ Thomas PI, Memphis, TN 38105 USA.
}

${ }^{3}$ Department of Cell Biology, Harvard Medical School, 200 Longwood Ave, Boston, MA 02115, USA.

4Program in Cellular and Molecular Medicine, Boston Children's Hospital, 200 Longwood Ave, Boston, MA 02115, USA.

${ }^{5}$ Department of Pediatrics, Harvard Medical School, 200 Longwood Ave, Boston, MA 02115, USA.

\footnotetext{
${ }^{*}$ Correspondence to: hessh@janelia.hhmi.org, betzige@janelia.hhmi.org.

\Current Address: Alnylam Pharmaceuticals, 300 Third Street, Cambridge, MA 02142

‡Current Address: Electron Microscopy Resource Center, The Rockefeller University, 1230 York Avenue, New York, NY 10065

†Current Address: Division of Gene Regulation, The Netherlands Cancer Institute, Plesmanlaan 121, Amsterdam, 1066 CX, The Netherlands

\# These authors contributed equally to this work.

Author contributions:

D.P.H., G.S., E.B., and H.F.H. supervised the project and wrote the manuscript with input from all coauthors. D.P.H. and G.S. designed and built the cryogenic optical microscope with input from E.B. and H.F.H. and performed all characterization experiments. D.P.H. and G.S. conducted all optical measurements. C.S.X optimized and adapted the FIB-SEM instruments. C.S.X., S.P., and D.P. conducted all EM experiments. D.E.M. created the instrument control software for the cryo-microscope. J.A.B. customized BigWarp. M.F. and K.S. prepared samples for Fig. 1-3 and the associated supplemental material. C.-L.C and L.W. prepared samples for Fig. 4 and 5, respectively. D.J.S. prepared all granule neuron samples and led related experiments. D.R.S., K.R.C. and D.J.S. performed LLSM and LLSM-SIM experiments. M.F., G.S., and D.P.H. froze specimens. G.S., H.A.P., and N.I. prepared all specimens for EM. D.P.H. and G.S. processed and analyzed all data except for that presented in Fig. 7 and the associated supplemental material which was done by K.R.C., D.R.S., A.S., and D.J.S. D.P.H. and G.S. produced all figures and movies except Fig. S18-S24, Movie S5, and the last part of Movie 6, which were produced by K.R.C. and D.R.S. W.P. assisted in aligning the data in Fig. 5. J.L.-S. and T.K. aided the biological interpretation of the results in Fig. 4 and 5, respectively.

Competing interests:

E.B. has a financial interest in LLSM. E.B. and H.F.H. have a financial interest in SMLM.

Data and materials availability:

All data needed to evaluate the conclusions of this paper are present here or the supplementary materials. The total size of the raw data used here exceeds tens of terabytes. All data used in this paper is freely available upon reasonable request to those who provide a mechanism for facile data transfer.
}

Supplementary Materials:

Supplementary Notes 1 to 16

Figs. S1 to S49

Tables S1 to S2

Captions for Movies S1 to S7

Movies S1 to S7

References (97-142) 
${ }^{6}$ Neuroimaging Laboratory, St. Jude Children's Research Hospital, 262 Danny Thomas PI, Memphis, TN 38105 USA.

7Bioimage Analysis Core, St. Jude Children's Research Hospital, 262 Danny Thomas PI, Memphis, TN 38105 USA.

${ }^{8}$ Department of Molecular and Cell Biology, University of California, Berkeley, CA 94720, USA.

${ }^{9}$ Department of Physics, University of California, Berkeley, CA 94720, USA.

${ }^{10}$ Howard Hughes Medical Institute, Berkeley, CA 94720, USA.

${ }^{11}$ Helen Wills Neuroscience Institute, Berkeley, CA 94720, USA.

${ }^{12}$ Molecular Biophysics and Integrated Bioimaging Division, Lawrence Berkeley National Laboratory, Berkeley, CA 94720, USA.

\section{Abstract}

Within cells, the spatial compartmentalization of thousands of distinct proteins serves a multitude of diverse biochemical needs. Correlative super-resolution (SR) fluorescence and electron microscopy (EM) can elucidate protein spatial relationships to global ultrastructure, but has suffered from tradeoffs of structure preservation, fluorescence retention, resolution, and field of view. We developed a platform for three-dimensional cryogenic SR and focused ion beam milled block-face EM across entire vitreously frozen cells. The approach preserves ultrastructure while enabling independent SR and EM workflow optimization. We discovered unexpected proteinultrastructure relationships in mammalian cells including intranuclear vesicles containing endoplasmic reticulum associated proteins, web-like adhesions between cultured neurons, and chromatin domains subclassified based on transcriptional activity. Our findings illustrate the value of a comprehensive multimodal view of ultrastructural variability across whole cells.

\section{One sentence summary:}

Cryogenic super-resolution fluorescence and electron microscopy reveals protein-ultrastructure relationships across whole cells.

\section{Summary:}

Cells function by the compartmentalization of thousands of distinct proteins, but the nanoscale spatial relationship of many proteins to the overall intracellular ultrastructure remains poorly understood. Hoffman et al. combined cryogenic super-resolution fluorescence microscopy and focused ion beam milling scanning electron microscopy to visualize protein-ultrastructure relationships in three dimensions (3D) across whole cells. The fusion of the two imaging modalities enabled positive identification and 3D segmentation at $8 \mathrm{~nm}$ isotropic sampling of morphologically complex structures within the crowded intracellular environment and revealed unexpected relationships, including a web-like protein adhesion network correlated to membrane roughness between juxtaposed cerebellar granule neurons.

Electron microscopy (EM) has revealed an intricate world inside eukaryotic cells (1), spatially organized at all length scales from nanometer-sized molecular assemblies to cell- 
spanning structures such as actin stress fibers and microtubules. However, even within different regions of the cell, there are notable differences in the structure of individual components, such as nuclear chromatin organization (2) or the morphology of the endoplasmic reticulum (ER), which is highly convoluted and compact in the perinuclear region, yet sparsely reticulated in lamellipodia (1). Thus, a comprehensive picture of cellular organization requires nanometer-level three-dimensional (3D) imaging of whole cells.

While cryogenic (cryo)-EM / tomography offers sub-nanometer 3D resolution (3), it is limited to sparse deposits of extracted macromolecules, cellular sections of sub-micron thickness (4-7), or thin lamella sculpted with cryo focused ion beam (FIB) milling $(8,9)$. In contrast, serial FIB ablation and imaging of the exposed face of resin-embedded specimens by scanning electron microscopy (FIB-SEM) routinely achieves $8 \mathrm{~nm}$ isotropic 3D sampling $(10-12)$ not possible with traditional 3D EM by diamond knife serial array $(13,14)$ or block face sectioning (15). However, EM produces grayscale images in which the unambiguous identification and 3D segmentation of many subcellular structures can be challenging, and where the distributions of specific proteins can rarely be identified.

In response, correlative light and electron microscopy (CLEM) techniques have been developed that combine the global contrast and high resolution of EM with the molecular specificity of fluorescence microscopy $(16,17)$. With the advent of super-resolution (SR) microscopy (18), such techniques now offer a closer match in resolution between the two modalities (table S1 and supplementary note 1), allowing specific molecular components to be visualized at nanoscale resolution in the context of the crowded intracellular environment. However, SR/EM correlation often involves tradeoffs in sample preparation between the retention of fluorescent labels, sufficiently dense heavy metal staining for high contrast EM, and faithful preservation of ultrastructure, particularly when chemical fixation is used (1922).

Here we describe a pipeline (fig. S1) for correlative cryo-SR/FIB-SEM imaging of whole cells designed to address these issues. Specifically, cryogenic, as opposed to room temperature, SR performed after high pressure freezing (HPF), allowed us to use a standard EM sample preparation protocol without compromise. We correlated cryogenic 3D structured illumination (SIM) and single molecule localization (SMLM) SR image volumes revealing protein specific contrast with 3D FIB-SEM image volumes containing global contrast of subcellular ultrastructure. The SR modality highlights features not readily apparent from the EM data alone, such as exceptionally long or convoluted endosomes, and permits unique classification of vesicles of like morphology, such as lysosomes, peroxisomes, and mitochondrial-derived vesicles. Cell-wide 3D correlation also reveals unexpected localization patterns of proteins, including intranuclear vesicles positive for an ER marker, intricate web-like structures of adhesion proteins at cell-cell junctions, and heterogeneity in euchromatin or heterochromatin recruitment of transcriptionally-associated histone H3.3 and heterochromatin protein 1a (HP1a) in the nuclei of neural progenitor cells as they transition into differentiated neurons. More generally, whole cell cryo-SR/FIB-SEM can reveal compartmentalized proteins within known subcellular components, help discover new subcellular components, and classify unknown EM morphologies and their roles in cell biology. 


\section{Cryogenic SR below 10K: motivations and photophysical characterization}

To avoid artifacts associated with chemical fixation (fig. S2), our pipeline begins with cryofixation via $\operatorname{HPF}(23,24)$ of whole cells cultured on $3 \mathrm{~mm}$ diameter, $50 \mu \mathrm{m}$ thick sapphire disks (supplementary note 2). Unlike plunge freeze methods, HPF reliably freezes specimens up to 200 microns thick $(21,23,25,26)$ in their entirety within vitreous ice in milliseconds, providing an exact snapshot of subcellular ultrastructure (fig. S3, movie S1). Each sapphire disk provides an optically flat and transparent back surface for aberration-free SR imaging, along with the high thermal conductivity needed to minimize specimen heating and potential ice re-crystallization under the intense $\left(\sim \mathrm{kW} / \mathrm{cm}^{2}\right)$, long-lasting illumination used during SMLM. Frozen specimens are inspected, cleaned (movie S2), and loaded onto a solid copper sample holder (fig. S4) in a covered, liquid nitrogen $\left(\mathrm{LN}_{2}\right)$ cooled preparation chamber (fig. S5) before transfer through a load lock to an evacuated optical cryostat modified for SR imaging (fig. S6).

Cryo-SR increases fluorophore photostability (27). This allowed us to achieve the high photon counts required for precise single molecule localization, despite the modest numerical aperture (NA 0.85) we were compelled to use in order to image through the cryostat window, vacuum, and sapphire substrate (fig. S1 and supplementary note 3 ). This along with a high reactivation efficiency under $405 \mathrm{~nm}$ illumination $(28,29)$ allowed us to acquire multicolor SIM/SMLM images of the same cells without substantial photobleaching. This, in turn, enabled SIM/SMLM correlation in three or more colors (movie S3) and allowed us to quickly image and assess many cells across the substrate by 3D SIM. We could then concentrate on the best candidates for much slower, higher resolution imaging by 3D SMLM.

Most cryo-SR systems to date operate with $\mathrm{LN}_{2}$ cooling near $77 \mathrm{~K}(7,27,29-34)$. However, we opted for a liquid helium (LHe) cooled microscope, which allowed us to explore photophysics at any temperature down to $8 \mathrm{~K}$ (supplementary note 4). In particular, we exploited a sharp increase in the lifetime of a dark state $\mathrm{D}_{1}$ for many fluorescent molecules with decreasing temperature (fig. S7) that allowed them to be shelved efficiently for long periods. Such shelving has important implications for SMLM, because it dictates the dynamic contrast ratio (DCR) defined by the time a given molecule is OFF and shelved in the dark state normalized to the time it is $\mathrm{ON}$ and cycling between singlet states $\mathrm{S}_{0}$ and $\mathrm{S}_{1}$ (fig. S7) to emit light. Molecules with high DCR can be expressed at higher density, creating SMLM images of higher fidelity and resolution, with less chance of spontaneous overlap of the diffraction spots from multiple molecules that would otherwise hinder precise localization.

We measured (Fig. 1A) the DCR of six different fluorophores at both $8 \mathrm{~K}$ and $77 \mathrm{~K}$ from the ON/OFF blinking behavior of isolated single molecules (fig. S8 and supplementary note 4a). In addition, we compared (Fig. 1B) their static contrast ratios (SCR, defined by the ratio of their signal in the ON state to their local background, fig. S9), which must also be high for precise localization, during SMLM imaging of densely labeled mitochondria (Movie 1, fig. S9, and supplementary note $4 \mathrm{~b}$ ). DCR and SCR tended to increase with shorter emission wavelengths, making such fluorophores better suited to high quality SMLM imaging (Fig. 
1C). SCR also often improved at lower temperature (Fig. 1B). These trends are consistent with the photophysical argument that the dark state lifetime should increase with increasing energy from $D_{1}$ to $S_{0}$, normalized to the thermal energy. In particular, we observed substantial gains in the SCR and DCR of JF525 (35) when operating with LHe which, in conjunction with mEmerald, enabled high quality two color SMLM of densely labeled structures. However, if only cryo-SIM and/or single color cryo-SMLM is needed, or if further study uncovers fluorophores spectrally distinct from mEmerald that work just as well at $77 \mathrm{~K}$, then operation with $\mathrm{LN}_{2}$ may prove sufficient.

To compare the relative merits of these labels for cryo-SMLM, we imaged two U2OS cells, targeting the ER membrane with mEmerald (green) and the mitochondrial outer membrane with Halo-JF525 (magenta) (36) or vice versa (Fig 1D). While both labels produced high density, high precision SMLM images of both targets, the Halo-JF525 images exhibited numerous bright puncta in both cases (Fig. 1D, fig. S10B). Although these may result from aggregation of Halo-tagged proteins, the presence of similar puncta in cryo-SMLM images of the ER obtained via SNAP (37) or CLIP (38) tag targeting of JF525 (fig. S10C, D) suggest that they arise from a subset of extremely long-lived JF525 molecules that undergo numerous switching cycles. Indeed, the long persistence of JF525 and other labels at 8K necessitated a fresh, data-driven approach (fig S11 and supplementary note 5b) to the problem of correctly assigning and integrating the multiple photon bursts from each molecule. Even so, in all cases mEmerald yielded images probably more reflective of the true molecular distribution.

\section{Cell-wide 3D correlation of cryo-SR with FIB-SEM}

A key advantage of our pipeline is that inserting the cryo-SR step between cryofixation and freeze substitution / staining for FIB-SEM allowed us to decouple the sample preparation protocols for the two imaging modalities. This avoids the tradeoffs of fluorescence retention, dense heavy metal staining, and ultrastructure preservation of resin-embedding based protocols (39-42). Furthermore, it allowed us to rapidly ( $20 \mathrm{~min})$ survey hundreds of cells across the sapphire disk (fig S12A), identify those of promising morphology and expression levels. We could then inspect these further at higher resolution by 3D cryo-SIM ( $5 \mathrm{~min} / \mathrm{cell} /$ color), and select the very best ones of these for the $\sim 1-2$ days/cell/color required for 3D cryo-SMLM and 10-15 days/cell needed for EM sample preparation and FIB-SEM imaging.

Thus, after cryo-SR imaging, we removed the frozen, disk-mounted specimens from the cryostat (fig. S12B) and processed them (supplementary note 6) via freeze substitution (1921), heavy metal staining, and embedding in Eponate 12 resin (fig S12C). After coarse trimming of the resin block and removal of the sapphire disk, we re-embedded the remaining tab in Durcupan, imaged it with x-rays (fig. S12D), and correlated this to the original diskwide view (fig S12E) to identify the region (typically $100 \times 100 \times 10 \mu \mathrm{m}$ ) containing the cells of interest imaged previously with cryo-SR. Additional microtome trimming isolated this region (fig. S12F-I), which we then imaged at $4-8 \mathrm{~nm}$ isotropic voxels in 3D by FIBSEM (12). 
To exploit the full potential of correlative microscopy, the different imaging modalities need to be mutually registered to the level of their spatial resolution. Given the high resolution of both cryo-SMLM and FIB-SEM, and our desire to extend their correlation across whole cells in 3D, registration to this level is challenging. For example, slight magnification differences or deviations from ideal flat field and rectilinear imaging coupled with potentially non-uniform FIB milling increase registration errors quickly with increasing field of view. Furthermore, freeze substitution and resin embedding introduce nonlinear and spatially inhomogeneous sample deformations (arrows, Fig. 2A) (43) between the cryo-SR and FIB-SEM imaging steps that have a substantial non-linear component requiring deformable registration to achieve alignment to this level of accuracy.

Taking advantage of our protocol, we densely labeled ubiquitous intracellular organelles such as the ER and mitochondria that could be readily identified in both the cryo-SMLM and FIB-SEM data and used them as landmarks (e.g., Fig. 2B, fig. S13) to register the EM to the SR across the cellular volume (supplementary note 7), using the software package BigWarp (44). To quantify the accuracy of this correlation, we independently measured the deformation fields $\overrightarrow{D F}_{E R}$ and $\overrightarrow{D F}_{\text {mito }}$ from only ER or mitochondrial landmarks, respectively, after aligning these color channels to one another using fluorescent bead fiducial markers. Because $\overrightarrow{D F}_{E R}$ and $\overrightarrow{D F}_{\text {mito }}$ represent independent estimates of the underlying sample deformation, the correlation accuracy $\varepsilon$ is given by $\left|\overrightarrow{D F}_{E R}-\overrightarrow{D F}_{\text {mito }}\right| / \sqrt{2}$ (supplementary note 7). Over a field of view covering the majority of two cells (pink surface, Fig. 2A), we measured a median $\varepsilon$ of $89 \mathrm{~nm}$ (Fig. 2C), whereas in a small peripheral region (red box, Fig. 2A), the median $\varepsilon$ was $27 \mathrm{~nm}$. The difference in $\varepsilon$ between these two regions of the same sample may be attributable to a higher density of landmarks within the peripheral region, tighter physical constraint on the differential motion between organelles due to the thinness of this region, or greater accuracy in landmark displacement measurement when the sample thickness becomes less than the axial localization precision. Regardless, spatial maps of $\varepsilon$ (fig. S14) give a local estimate of the length scale to which spatial relationships between features seen by the two imaging modalities can be reliably inferred.

\section{Correlative cryo-SR/FIB-SEM reveals vesicle identities and their morphological diversity}

Using our correlative pipeline, we imaged two neighboring COS-7 cells (Fig. 3, Movie 2) transiently expressing mEmerald-ER3, a luminal ER marker, and HaloTag-TOMM20 conjugated to JF525 to label the mitochondrial outer membrane. Both the resulting volume rendering (Fig. 3A) and axial or transverse orthoslices (Figs. 3B-M, fig. S15) demonstrate accurate 3D correlation of the cryo-SMLM and FIB-SEM data, high labeling density, and faithful ultrastructure preservation throughout the $\sim 5000 \mu \mathrm{m}^{3}$ cellular volume within the field of view.

The data also immediately illustrate the power of cryo-SR and FIB-SEM correlation. For example, clusters of ER3 seen by cryo-SMLM to dot ER tubules (orange arrows, Fig. 3B) 
might easily be dismissed as artifacts of labeling or fixation, but instead correlate (Fig. 3D) with varicosities in these tubules as seen by FIB-SEM (Fig. 3C). It is also immediately apparent by FIB-SEM that vesicles of various sizes are ubiquitous throughout the cell. However, these can come in many forms - peroxisomes, lysosomes, endosomes or, as identified by our correlation, TOMM20-positive vesicles (red arrows, Fig. 3H-J, fig. S16). Given their small ( $100-200 \mathrm{~nm})$ size and proximity to mitochondria, these may represent mitochondria derived vesicles (MDVs). MDVs are believed to play a key role in mitochondrial quality control by sequestering unfolded or oxidized mitochondrial proteins and transporting them to lysosomes or peroxisomes for degradation (45). There remain, however, many questions about what proteins regulate these processes and where they are distributed.

Our data also revealed three instances of intranuclear vesicles, again 100-200 nm size, positive for the ER lumen protein ER3 (left inset orthoslices, Fig. 3A; correlation examples 119 and 164, fig. S15). In dividing somatic cells, the nuclear membrane (NM) breaks down at prometaphase and NM proteins are dispersed within the ER, which remains continuous throughout mitosis (46). The NM then begins to reassemble in anaphase when ER-like cisternae contact the chromatids of the daughter cells, and NM proteins become immobilized there (46-49). Thus, one possibility is that ER lumen-positive intranuclear vesicles in interphase are the remnants of such contacts that do not completely return to the extranuclear ER after the NM is fully re-established. Alternatively, a small fraction of the total ER volume might be disrupted into vesicles during its rearrangement in mitosis and become similarly trapped when the NM reforms.

Another important class of vesicles in cells are peroxisomes, which catabolize long chain fatty acids via $\beta$-oxidation and reduce reactive oxygen species such as hydrogen peroxide (50). Peroxisomes can adopt a variety of sizes, shapes, and distributions depending on cell type and environment $(50,51)$. Accurately capturing these morphologies and their spatial relationship to other organelles can be difficult with traditional chemical fixation and EM staining protocols against their enzymatic contents $(52,53)$. Furthermore, serial section transmission EM or mechanically sectioned block face EM (54) lack the axial resolution to precisely measure morphological parameters at the sub-100 nm level, whereas cryo-FIBSEM (55) or cryo-EM tomography lacks the field of view to explore more than a small fraction of the total cellular volume.

We used cryo-SMLM / FIB-SEM to image and semi-automatically segment (supplementary note 8) 466 mature peroxisomes across two entire vitreously frozen HeLa cells expressing mEmerald tagged to the peroxisomal targeting sequence SKL, and Halo/JF525-TOMM20 to mark mitochondria (Fig. 4, Movie 3, and fig. S17). Independent two-channel SR/EM registration revealed a correlation accuracy (median $\varepsilon$ ) of $85 \mathrm{~nm}$ (fig. S14C, D). Peroxisomes with volumes smaller than $0.01 \mu \mathrm{m}^{3}$ always assumed nearly spherical shapes presumably to minimize their surface area under the influence of surface tension (e.g., Fig. 4A, H). Increasingly irregular shapes such as plates (Fig. 4B), cups (Fig. 4C) or hollow spheres (Fig. 4D) formed with increasing volume (lower rows, fig. S17). This may serve to regulate reaction kinetics rates within peroxisomes (56). Some irregularly shaped peroxisomes were in close proximity to other organelles or as part of multi-organelle 
assemblies (Fig. 4E-G), consistent with 3D observations in live cells (57). These assemblies may facilitate the transfer of cargo between organelles responsible for distinct and possibly incompatible biochemical processes (57), such as the sequential breakdown of fatty acids between peroxisomes and mitochondria (58).

Finally, we explored the endolysosomal pathway, the compartments of which are notoriously sensitive to artifacts of fixation or protein overexpression (59-61). We used correlative cryoSIM/FIB-SEM to image transferrin (Tfn)-containing endolysosomal compartments in a SUM-159 cell previously incubated for $30 \mathrm{~min}$ in media containing Alexa Fluor 647conjugated Tfn (Fig. 5A, beginning of Movie 4). The density of labeled compartments was low enough to assign each discrete SIM feature (inset, Fig. 5A) to a single structure as seen by FIB-SEM, and then render each such compartment with $8 \mathrm{~nm}$ isotropic voxels. Despite its much lower resolution, SIM was essential to identify which compartments in the FIBSEM data represented endolysosomes and to spot the many such structures of extremely convoluted morphology in the crowded intracellular environment that were not readily apparent by FIB-SEM alone. These included elongated tubules (magenta, Figs. 5B-E) that likely represent recycling endosomes, highly corrugated endosomes (Figs. 5B, D, right), and early endosomes with protruding tubules of $50 \mathrm{~nm}$ width possibly associated with retromers (62) of sub-50 nm width. Given that cryo-SIM is much faster than cryo-SMLM and can use a wide variety of spectrally distinct labels, it can be a broadly useful tool in its own right to guide the 3D segmentation of dense FIB-SEM data and ensure the correct identification of specific subcellular features.

\section{Molecular underpinnings of ultrastructural specialization in neuronal cell- to-cell adhesions}

Cell-to-cell adhesions mediate cell migration, nucleate cell polarity and spur communication between individual cells in multi-cellular organisms $(63,64)$. While the molecular context and ultrastructure of cellular adhesions to rigid artificial substrates are well characterized $(65,66)$ those between cells in complex 3D environments are not. Neuronal adhesions are crucial for brain development, playing an integral role in sorting neurons based on their maturation status $(67,68)$, forming the laminar structure of the brain $(69,70)$, and ultimately promoting the complex neuronal interactions that drive circuit morphogenesis (71).

However, they have been difficult to study because they are disrupted by chemical fixation (19-21), and because 3D geometries of neuronal contacts require isotropic 3D-EM and highresolution LM.

We used cryo-SIM to visualize transiently expressed junctional adhesion molecule (JAM)-C $(67,69,72)$, a tight-junction component, fused to JF549i-conjugated SNAP $(35,73)$, and 2xmVenus-drebrin, a cytoplasmic actin-microtubule crosslinker protein (74), in cryofixed mouse cerebellar granule neurons (CGNs) (Fig. 6A, Movie 5). The JAM-C defined adhesion between two labeled somas was not uniform at their shared membrane contact zone (CZ) (Fig. 6B) but formed a web-like structure with drebrin preferentially associated with the edges of the JAM-C regions. 
To determine if these protein distributions correlated with membrane ultrastructure at the CZ, we imaged the same cells by FIB-SEM (Fig. 6C). The density of heavy metal staining at the PM was also nonuniform (Fig. 6D), with the densest staining correlating perfectly with JAM-C (compare Figs. 6B, D and G). Moreover, the densely stained PM was less curved than the electron lucent PM. To quantify this, we segmented the PM within the $\mathrm{CZ}$ into high and low electron density regions (Figs. 6F, I), and then calculated the curvedness (supplementary note 9) in each (Figs. 6H and I). The low-density PM was 2.3 times more curved than the high-density, JAM-C rich PM.

While the smooth nature of the adhesion as defined by JAM-C is expected because of the mechanical tension induced by the juxtacrine interaction (75-77), the fact that the adhesion does not comprise the whole contact area between these two cells is not. Furthermore, the enrichment of drebrin in the regions adjacent to JAM-C contrasts with the laminar stacking of adhesion-associated cytoskeletal adaptor proteins found in focal or cadherin-based adhesions on glass $(65,66)$.

\section{Chromatin domains and their reorganization during neuronal differentiation}

In addition to adhesion, CGNs provide an excellent model system to study the cell biological underpinnings of neural development owing to their strongly stereotyped developmental programs as they differentiate from cerebellar granule neuron progenitors (GNPs) (78). Intrinsic to this process is the 3D structural reorganization of their nuclear chromatin domains $(79,80)$. To explore this in detail, we first used 3D live cell lattice light sheet microscopy (LLSM) (81). Flow sorted GNPs expressing the EGFP-Atoh1 marker of the GNP-state $(82,83)$ possessed significantly larger nuclei than terminally differentiated CGNs (Figure 7A, B, and supplementary note 10). Moreover, longitudinal LLSM live-imaging revealed that GNPs rapidly condense their nuclei to the size of CGNs while Atoh1-EGFP expression fades (Figure 7A, B, movie S4).

To uncover the intricate 3D transformations in nuclear architecture that accompany nuclear condensation during GNP differentiation, we then applied cryo-SIM to image a cohort of 7 GNPs and 9 CGNs. These collectively contained $>2000 \mu \mathrm{m}^{3}$ of the nuclear domain reference proteins mEmerald tagged heterochromatin protein 1 alpha (HP1a), a prototypical heterochromatin marker (84), and JF525-conjugated SNAP-Histone 3.3 (H3.3), a replacement histone subunit that is loaded on transcriptionally active nucleosomes (85) (Fig. 7C, G, top, fig. S18). We followed this with FIB-SEM imaging and segmentation of the resulting data (86) according to the classic EM definitions of compacted heterochromatin, open euchromatin and nucleoli (2) (Fig. 7C, G, bottom). While GNP and CGN nuclei possessed similar total nuclear volumes of compacted heterochromatin $\left(\mathrm{GNP}=47 \pm 2 \mu \mathrm{m}^{3}\right.$, CGN $=45 \pm 3 \mu \mathrm{m}^{3}$ ), GNP nuclei had a significantly higher total nuclear volume of euchromatin $\left(\mathrm{GNP}=84 \pm 8 \mu \mathrm{m}^{3}, \mathrm{CGN}=61 \pm 6 \mu \mathrm{m}^{3}\right)$ that accounted for a significant fraction of the size differential with CGNs (Fig. 7K).

Registering the cryo-SIM data onto the FIB-SEM results (Fig. 7D-F, H-J, Movie 6, figs. S19-S21, movie S5, and supplementary note 11) allowed us to subclassify these classical EM chromatin domains based on their correlation to HP1a or H3.3 (figs. S22, S23, and 
supplementary note 12). Such correlation revealed variations in these chromatin domains linked to neuronal differentiation that are not discernable by ultrastructure alone, including classical compacted heterochromatin domains with alternating layers HP1a and H3.3 (Figure 7J). Indeed, while FIB-SEM showed little difference in the absolute volume of compacted heterochromatin before and after differentiation, correlation with cryo-SIM revealed that CGN nuclei had $\sim 50 \%$ more normalized nuclear volume of HP1a loaded heterochromatin than GNPs (GNP $=8 \pm 1 \%, \mathrm{CGN}=12 \pm 2 \%$, Fig. 7L). Moreover, surface area to volume measurements showed HP1a loaded heterochromatin became substantially more compact during nuclear condensation (fig. S23A).

Analysis of $\mathrm{H} 3.3$ relationships to heterochromatin and euchromatin also revealed substantial differences between GNP and CGN nuclei. While both GNPs and CGNs had similar amounts of H3.3-loaded euchromatin ( $\mathrm{GNP}=27 \pm 4 \%, \mathrm{CGN}=32 \pm 3 \%$, Fig. 7L) indicative of transcriptionally active regions, GNPs had 50\% more normalized nuclear volume of a H3.3free form of euchromatin than did CGNs (GNP $=29 \pm 4 \%, C G N=20 \pm 2 \%$, Fig. 7L). Live cell LLSM operating in the higher resolution SIM mode revealed that these large H3.3-free voids in GNP nuclei contain mEmerald-cMAP3, a marker of H3K27me3 and H3K4me3loaded poised chromatin (87) suggesting that groups of poised genes are organized in a region-specific fashion in neural progenitors (fig. S24, movie S6, and supplementary note 13).

GNP differentiation into CGNs also resulted in the unexpected accumulation of $\mathrm{H} 3.3$ in heterochromatin nearly twice as abundant in CGNs as GNPs (GNP $=13 \pm 1 \%, \mathrm{CGN}=$ $22 \pm 3 \%$, Fig. 7L). Like classical HP1a-loaded heterochromatin, H3.3-heterochromatin also underwent compaction during CGN differentiation (fig. S23C). The presence of a large fraction of H3.3-loaded heterochromatin in differentiated neurons was surprising given H3.3-loaded heterochromatin species are abundant in pluripotent embryonic stem cells (ESCs) but have not been observed in most of their somatic cell derivatives $(88,89)$. Furthermore, LLS-SIM revealed that H3.3-loaded heterochromatin is likely not due to H3.3 recruitment to telomeres or centromeres as has been reported for ESCs (fig. S24B).

Finally, heterochromatin subdomains exhibited spatially distinct organization patterns depending on whether they were loaded with HP1a or H3.3 (fig. S22A, movie S7). Additional analysis based on the density of heavy metal staining in a correlated 4 nm FIBSEM data set revealed that H3.3-heterochromatin was less densely packed then HP1aheterochromatin in CGN nuclei, showing that molecularly defined heterochromatin subdomains are not only spatially distinct at the level of the whole nucleus but are also morphologically distinct at the ultrastructural level (Fig. S22B).

\section{Discussion}

Much of what we know about the structural and functional organization of the cell at the nanoscale comes from a synthesis of the findings of EM, biochemistry, and molecular biology. Although this synthesis has proved powerful, fusing the insights from these disparate methods necessarily involves developing models, and therefore possible biases, of how specific proteins are spatially distributed in relation to the EM ultrastructure that bear 
closer examination. Correlative cryo-SR/FIB-SEM enables such examination by combining two complementary datasets, often revealing unanticipated protein localization patterns or ultrastructural morphologies at variance with such models. At the same time the approach enables the discovery of new subcategories of functionally distinct subcellular structures that appear morphologically similar by either SR or EM alone. As such, it provides observations upon which more refined models can be developed in a way mutually consistent with the findings of SR, EM, live imaging, and biochemistry.

Of course, the value of cryo-SR/FIB-SEM to this enterprise depends on the extent to which it reveals the native ultrastructure of the cells it images, and the extent to which these cells are representative of the normal physiological state of their class. We designed our pipeline with these goals in mind. HPF immediately followed by cryo-SR imaging of cells in vitreous ice without any intervening chemical modification ensures that a faithful, unperturbed snapshot of the cell is captured, and allows SR and EM sample preparation protocols to be decoupled and independently optimized. Widefield cryo-fluorescence imaging to rapidly survey hundreds of cells, followed by higher resolution inspection of likely candidates by multicolor 3D cryo-SIM at a few minutes per cell ensures that only those cells of physiological morphology, or ones in a specific desired physiological state (e.g., (90)) are considered for time-intensive cryo-SMLM and FIB-SEM. Lastly, freeze substitution provides excellent preservation of native ultrastructural detail, while subsequent whole-cell 3D FIB-SEM gives a comprehensive picture of subcellular components across all regions of the cell, at 4 or $8 \mathrm{~nm}$ isotropic voxels not possible by serial section transmission EM or mechanically sectioned serial block face EM.

That being said, cryo-EM tomography of thin lamellae excavated from whole cells by cryoFIB $(8,9)$ offers molecular resolution without any risk of ultrastructural perturbation by heavy metal staining and resin embedding. Given, however, that the lamellar volume is typically only a small fraction of the entire cellular volume, many structures of interest will be missed entirely, and those that are seen may not exhibit the same morphology as in other regions of the cell. Thus, FIB-SEM and cryo-EM tomography are complementary and developing a pipeline to do both in conjunction with cryo-SR would be a worthwhile endeavor.

Indeed, the unique ability of FIB-SEM to image whole cells and tissues at 4-8 nm isotropic voxels over volumes as large as $10^{7} \mu \mathrm{m}^{3}$ makes it an ideal tool to map in toto the 3D ultrastructural relationships in living systems. However, to unlock its full potential, robust automated identification and segmentation of specific intracellular features of interest is required, ideally in relationship to neighboring structures with which such features might interact. This remains challenging to accomplish at scale, given the magnitude of the data involved (e.g., 100 GB in Fig. 7 and 19.5 TB in (12)), the diversity, spatial density, and conformational complexity of intracellular compartments, and the monochromatic nature of the data. Cryo-SR can play an important role in the development of scalable segmentation, both in the validation of training sets for machine learning, and in confirmation of the resulting segmented outputs. 
We can also envision a number of possible improvements to our pipeline. First, live cell imaging immediately prior to freezing would allow correlation of dynamics to ultrastructure (61), refine selection to cells of physiological behavior, and enable pharmacological, optogenetic, or other perturbations to be applied. However, the logistics for rapid and noninvasive transition from live imaging to the frozen state will require substantial technological development. Second, an extension of cryo-SR/FIB-SEM to specimens such as small gene-edited organisms or organoids that are more physiologically relevant than the isolated adherent cells with ectopically expressed markers presented here should be feasible within the $200 \mu \mathrm{m}$ thickness limit for HPF by incorporating adaptive optics for aberrationfree deep imaging. Third, the axial resolution of both cryo-SIM and cryo-SMLM could be improved $\sim 5-10 \times$ by designing a dual window cryostat using opposed objectives and coherent detection, such as in $\mathrm{I}^{5} \mathrm{~S}$ (91) and iPALM (92). A next generation pipeline combining these improvements could prove an even more powerful discovery platform to link 3D subcellular dynamic processes in cells, small whole organisms, and acute tissue sections to the nanoscale spatial distribution of the proteins driving these processes, all in the context of the global intracellular ultrastructure. However, even in its current form, our cryoSR/FIB-SEM system can address a broad range of biological questions and is available to outside users wanting to do so (93).

\section{Materials and methods}

\section{Preparation of vitrified samples}

Specimens were cultured on $3 \mathrm{~mm}$ diameter, $50 \mu \mathrm{m}$ thick sapphire disks (Nanjing CoEnergy Optical Crystal Co., Ltd, custom order, supplementary note 2) before cryofixation with a Wohlwend Compact 2 high pressure freezer. Sample-specific protocols and plasmid maps can be found in supplementary note 14 .

\section{Cryogenic light microscopy}

To optically image vitrified samples at diffraction-limited resolution and beyond, they must be maintained below $125 \mathrm{~K}$ to avoid de-vitrification (94), and present a clean, optically flat surface for aberration-free imaging. To achieve these ends, we built our microscope around a modified commercial liquid helium flow cryostat (Janis Research Company, ST-500, supplementary note 3, fig. S6) and imaged cells plated on sapphire coverslips (supplementary note 2) through the opposite surface, after clearing this surface of residual ice in a custom cryo-preparation chamber (fig. S5 and movie S2, and supplementary note 2). We transferred samples from cold storage to the imaging cryostat using custom tools and procedures adapted to a commercial cryogenic vacuum transfer system (Quorum Technologies, PP3010T, fig. S6, and supplementary note 3). SIM images were processed as previously described (95) and SMLM processing is described in supplementary note 15.

\section{EM sample preparation}

Following optical imaging, samples were transferred back to cryo-storage before being freeze-substituted, resin embedded, and re-embedded (supplementary notes 6B, 6C). Desired regions of interest (ROIs) were identified in the plasticized specimens (fig. S12) using an 
XRadia 510 Versa micro X-Ray system (Carl Zeiss X-ray Microscopy, Inc.) and then trimmed to expose small ( 100 x $100 \times 60 \mu \mathrm{m})$ stubs (supplementary note $6 \mathrm{D}$ ).

FIB-SEM imaging

Standard ( $8 \times 8 \times 8 \mathrm{~nm}^{3}$ isotropic voxel) FIB-SEM datasets were generated using a customized Zeiss Merlin crossbeam system previously described (12) and further modified as specified in supplementary note 16 . The SEM image stacks were acquired at $500 \mathrm{kHz} /$ pixel with an $8 \mathrm{~nm} x-y$ pixel using a $2 \mathrm{nA}$ electron beam at $1.2 \mathrm{kV}$ landing energy for imaging and a $15 \mathrm{nA}$ gallium ion beam at $30 \mathrm{kV}$ for FIB milling. $4 \times 4 \times 4 \mathrm{~nm}^{3}$ voxel datasets were generated using a similarly customized Zeiss GeminiSEM 500-Capella Crossbeam system. The block face was imaged by a $250 \mathrm{pA}$ electron beam with $0.9 \mathrm{kV}$ landing energy at $200 \mathrm{kHz}$. The final image stacks were registered using a SIFT (96) based algorithm.

\section{Computing resources}

For most of the data analysis, except initial SMLM peak detection and fitting, we used a stand-alone Windows-10 x64 workstation with dual Xeon Gold 5122 CPUs (3.60 GHz) and $1 \mathrm{~TB}$ of RAM. For SMLM peak detection and fitting we used up to 256 nodes on the Janelia cluster.

\section{Supplementary Material}

Refer to Web version on PubMed Central for supplementary material.

\section{Acknowledgments:}

We thank L. Lavis, W. Legant, D. Li, L. Shao, C. Ott, N. Alivodej, Niraj Trivedi, Danielle Wong and K. Hayworth. We also thank the Shared Resource teams at Janelia for their skill and dedication in specimen handling and preparation and the Janelia Experimental Technologies team for their manufacturing expertise, in particular Bill Biddle and Bruce Bowers. We gratefully acknowledge the support of the Janelia Visitor Program. mEmerald-N1 and mEmerald-ER3 were a gifts from Michael Davidson (Addgene plasmid \#53976; http://n2t.net/addgene:53976; RRID:Addgene_53976 and Addgene plasmid \#54082; http://n2t.net/addgene:54082; RRID:Addgene_54082). D.P.H., G.S., C.S.X., M.F., D.E.M., H.A.P, N.I., J.A.B., S.P., D.P., K.S., C-L.C., J.L-S., E.B., and H.F.H were funded by the Howard Hughes Medical Institute (HHMI). L.W., W.P., and T.K. were funded by grants from Biogen, NIH R01 GM075252 and a MIRA NIH award GM130386 (to T.K.). K.R.C., D.R.S., A.S. and D.J.S. were funded by the American Lebanese Syrian Associated Charities (ALSAC) and by grants 1R01NS066936 and R01NS104029-02 from the National Institute of Neurological Disorders (NINDS). LLSM images were acquired at St. Jude Children's Research Hospital in the Department of Developmental Neurobiology Neuroimaging Laboratory.

\section{References and Notes:}

1. Fawcett DW, The cell (W. B. Saunders Co, Philadelphia, 2d ed., 1981).

2. Ou HD, Phan S, Deerinck TJ, Thor A, Ellisman MH, O’Shea CC, ChromEMT: Visualizing 3D chromatin structure and compaction in interphase and mitotic cells. Science. 357, eaag0025 (2017). [PubMed: 28751582]

3. Briggs JA, Structural biology in situ-the potential of subtomogram averaging. Current Opinion in Structural Biology. 23, 261-267 (2013). [PubMed: 23466038]

4. Al-Amoudi A, Chang J-J, Leforestier A, McDowall A, Salamin LM, Norlén LP, Richter K, Blanc NS, Studer D, Dubochet J, Cryo-electron microscopy of vitreous sections. The EMBO Journal. 23, 3583-3588 (2004). [PubMed: 15318169] 
5. Al-Amoudi A, Norlen LPO, Dubochet J, Cryo-electron microscopy of vitreous sections of native biological cells and tissues. Journal of Structural Biology. 148, 131-135 (2004). [PubMed: 15363793]

6. Alamoudi A, Studer D, Dubochet J, Cutting artefacts and cutting process in vitreous sections for cryo-electron microscopy. Journal of Structural Biology. 150, 109-121 (2005). [PubMed: 15797735]

7. Liu B, Xue Y, Zhao W, Chen Y, Fan C, Gu L, Zhang Y, Zhang X, Sun L, Huang X, Ding W, Sun F, Ji W, Xu T, Three-dimensional super-resolution protein localization correlated with vitrified cellular context. Scientific Reports. 5, 13017 (2015). [PubMed: 26462878]

8. Schaffer M, Mahamid J, Engel BD, Laugks T, Baumeister W, Plitzko JM, Optimized cryo-focused ion beam sample preparation aimed at in situ structural studies of membrane proteins. Journal of Structural Biology. 197, 73-82 (2017). [PubMed: 27444390]

9. Pfeffer S, Mahamid J, Unravelling molecular complexity in structural cell biology. Current Opinion in Structural Biology. 52, 111-118 (2018). [PubMed: 30339965]

10. Knott G, Marchman H, Wall D, Lich B, Serial Section Scanning Electron Microscopy of Adult Brain Tissue Using Focused Ion Beam Milling. J. Neurosci 28, 2959-2964 (2008). [PubMed: 18353998]

11. Narayan K, Subramaniam S, Focused ion beams in biology. Nature Methods. 12, 1021-1031 (2015). [PubMed: 26513553]

12. Xu CS, Hayworth KJ, Lu Z, Grob P, Hassan AM, García-Cerdán JG, Niyogi KK, Nogales E, Weinberg RJ, Hess HF, Enhanced FIB-SEM systems for large-volume 3D imaging. eLife. 6, e25916 (2017). [PubMed: 28500755]

13. Hayworth KJ, Kasthuri N, Schalek R, Lichtman JW, Automating the Collection of Ultrathin Serial Sections for Large Volume TEM Reconstructions. Microscopy and Microanalysis. 12, 86-87 (2006).

14. Bock DD, Lee W-CA, Kerlin AM, Andermann ML, Hood G, Wetzel AW, Yurgenson S, Soucy ER, Kim HS, Reid RC, Network anatomy and in vivo physiology of visual cortical neurons. Nature. 471, 177-182 (2011). [PubMed: 21390124]

15. Denk W, Horstmann H, Serial Block-Face Scanning Electron Microscopy to Reconstruct ThreeDimensional Tissue Nanostructure. PLOS Biology. 2, e329 (2004). [PubMed: 15514700]

16. de Boer P, Hoogenboom JP, Giepmans BNG, Correlated light and electron microscopy: ultrastructure lights up! Nature Methods. 12, 503-513 (2015). [PubMed: 26020503]

17. Hauser M, Wojcik M, Kim D, Mahmoudi M, Li W, Xu K, Correlative Super-Resolution Microscopy: New Dimensions and New Opportunities. Chem. Rev (2017), doi:10.1021/ acs.chemrev.6b00604.

18. Wegel E, Göhler A, Lagerholm BC, Wainman A, Uphoff S, Kaufmann R, Dobbie IM, Imaging cellular structures in super-resolution with SIM, STED and Localisation Microscopy: A practical comparison. Scientific Reports. 6, 27290 (2016). [PubMed: 27264341]

19. Keene DR, McDonald K, The ultrastructure of the connective tissue matrix of skin and cartilage after high-pressure freezing and freeze-substitution. J Histochem Cytochem 41, 1141-1153 (1993). [PubMed: 8331280]

20. McDonald KL, in Methods in Cell Biology, Goldstein LSB, Fyrberg EA, Eds. (Academic Press, 1994; http://www.sciencedirect.com/science/article/pii/S0091679X08609267), vol. 44, pp. 411444. [PubMed: 7707966]

21. McDonald K, Morphew M, Verkade P, Müller-Reichert T, in Electron Microscopy, Kuo J, Ed. (Humana Press, Totowa, NJ, 2007; http://www.springerlink.com/index/ 10.1007/978-1-59745-294-6), vol. 369 of Methods in Molecular Biology, pp. 143-173. [PubMed: 17656750]

22. Schnell U, Dijk F, Sjollema KA, Giepmans BNG, Immunolabeling artifacts and the need for livecell imaging. Nat Meth 9, 152-158 (2012).

23. Studer D, Humbel BM, Chiquet M, Electron microscopy of high pressure frozen samples: bridging the gap between cellular ultrastructure and atomic resolution. Histochem Cell Biol 130, 877-889 (2008). [PubMed: 18795316] 
24. Studer D, Zhao S, Chai X, Jonas P, Graber W, Nestel S, Frotscher M, Capture of activity-induced ultrastructural changes at synapses by high-pressure freezing of brain tissue. Nat. Protocols 9 , 1480-1495 (2014). [PubMed: 24874814]

25. Kiss JZ, Jr THG, Staehelin LA, Sack FD, Comparison of the ultrastructure of conventionally fixed and high pressure frozen/freeze substituted root tips ofNicotiana andArabidopsis. Protoplasma 157, 64-74 (1990). [PubMed: 11538077]

26. Dahl R, Staehelin LA, High-pressure freezing for the preservation of biological structure: Theory and practice. Journal of Electron Microscopy Technique 13, 165-174 (1989). [PubMed: 2685196]

27. Li W, Stein SC, Gregor I, Enderlein J, Ultra-stable and versatile widefield cryo-fluorescence microscope for single-molecule localization with sub-nanometer accuracy. Optics Express. 23, 3770 (2015). [PubMed: 25836229]

28. Schwentker MA, Parallelized Ground State Depletion (2007), (available at http://archiv.ub.uniheidelberg.de/volltextserver/7677/).

29. Tuijtel MW, Koster AJ, Jakobs S, Faas FGA, Sharp TH, Correlative cryo super-resolution light and electron microscopy on mammalian cells using fluorescent proteins. Scientific Reports. 9, 1369 (2019). [PubMed: 30718653]

30. Chang Y-W, Chen S, Tocheva EI, Treuner-Lange A, Löbach S, Søgaard-Andersen L, Jensen GJ, Correlated cryogenic photoactivated localization microscopy and cryo-electron tomography. Nat Meth 11, 737-739 (2014).

31. Kaufmann R, Schellenberger P, Seiradake E, Dobbie IM, Jones EY, Davis I, Hagen C, Grünewald K, Super-Resolution Microscopy Using Standard Fluorescent Proteins in Intact Cells under CryoConditions. Nano Lett 14, 4171-4175 (2014). [PubMed: 24884378]

32. Dahlberg PD, Sartor AM, Wang J, Saurabh S, Shapiro L, Moerner WE, Identification of PAmKate as a Red Photoactivatable Fluorescent Protein for Cryogenic Super-Resolution Imaging. J. Am. Chem. Soc (2018), doi:10.1021/jacs.8b05960.

33. Moser F, Pražák V, Mordhorst V, Andrade DM, Baker LA, Hagen C, Grünewald K, Kaufmann R, Cryo-SOFI enabling low-dose super-resolution correlative light and electron cryo-microscopy. PNAS 116, 4804-4809 (2019). [PubMed: 30808803]

34. Wang L, Bateman B, Zanetti-Domingues LC, Moores AN, Astbury S, Spindloe C, Darrow MC, Romano M, Needham SR, Beis K, Rolfe DJ, Clarke DT, Martin-Fernandez ML, Solid immersion microscopy images cells under cryogenic conditions with $12 \mathrm{~nm}$ resolution. Communications Biology. 2, 74 (2019). [PubMed: 30820469]

35. Grimm JB, Muthusamy AK, Liang Y, Brown TA, Lemon WC, Patel R, Lu R, Macklin JJ, Keller PJ, Ji N, Lavis LD, A general method to fine-tune fluorophores for live-cell and in vivo imaging. Nature Methods. 14, 987-994 (2017). [PubMed: 28869757]

36. Los GV, Encell LP, McDougall MG, Hartzell DD, Karassina N, Zimprich C, Wood MG, Learish R, Ohana RF, Urh M, Simpson D, Mendez J, Zimmerman K, Otto P, Vidugiris G, Zhu J, Darzins A, Klaubert DH, Bulleit RF, Wood KV, HaloTag: A Novel Protein Labeling Technology for Cell Imaging and Protein Analysis. ACS Chem. Biol 3, 373-382 (2008). [PubMed: 18533659]

37. Keppler A, Gendreizig S, Gronemeyer T, Pick H, Vogel H, Johnsson K, A general method for the covalent labeling of fusion proteins with small molecules in vivo. Nature Biotechnology. 21, 86 (2003).

38. Gautier A, Juillerat A, Heinis C, Corrêa IR, Kindermann M, Beaufils F, Johnsson K, An Engineered Protein Tag for Multiprotein Labeling in Living Cells. Chemistry \& Biology. 15, 128136 (2008). [PubMed: 18291317]

39. Kim D, Deerinck TJ, Sigal YM, Babcock HP, Ellisman MH, Zhuang X, Correlative Stochastic Optical Reconstruction Microscopy and Electron Microscopy. PLOS ONE. 10, e0124581 (2015). [PubMed: 25874453]

40. Peddie CJ, Domart M-C, Snetkov X, O’Toole P, Larijani B, Way M, Cox S, Collinson LM, Correlative super-resolution fluorescence and electron microscopy using conventional fluorescent proteins in vacuo. Journal of Structural Biology. 199, 120-131 (2017). [PubMed: 28576556]

41. Paez-Segala MG, Sun MG, Shtengel G, Viswanathan S, Baird MA, Macklin JJ, Patel R, Allen JR, Howe ES, Piszczek G, Hess HF, Davidson MW, Wang Y, Looger LL, Fixation-resistant 
photoactivatable fluorescent proteins for CLEM. Nature Methods. 12, 215-218 (2015). [PubMed: 25581799]

42. Johnson E, Seiradake E, Jones EY, Davis I, Grünewald K, Kaufmann R, Correlative in-resin superresolution and electron microscopy using standard fluorescent proteins. Scientific Reports. 5, 9583 (2015). [PubMed: 25823571]

43. Matsko N, Mueller M, Epoxy resin as fixative during freeze-substitution. Journal of Structural Biology. 152, 92-103 (2005). [PubMed: 16214372]

44. Bogovic JA, Hanslovsky P, Wong A, Saalfeld S, in 2016 IEEE 13th International Symposium on Biomedical Imaging (ISBI) (IEEE, Prague, Czech Republic, 2016; http://ieeexplore.ieee.org/ document/7493463/), pp. 1123-1126.

45. Sugiura A, McLelland G-L, Fon EA, McBride HM, A new pathway for mitochondrial quality control: mitochondrial-derived vesicles. The EMBO Journal. 33, 2142-2156 (2014). [PubMed: 25107473]

46. Burke B, Ellenberg J, Remodelling the walls of the nucleus. Nature Reviews Molecular Cell Biology. 3, 487 (2002). [PubMed: 12094215]

47. Anderson DJ, Hetzer MW, Reshaping of the endoplasmic reticulum limits the rate for nuclear envelope formation. The Journal of Cell Biology. 182, 911-924 (2008). [PubMed: 18779370]

48. Ellenberg J, Siggia ED, Moreira JE, Smith CL, Presley JF, Worman HJ, Lippincott-Schwartz J, Nuclear Membrane Dynamics and Reassembly in Living Cells: Targeting of an Inner Nuclear Membrane Protein in Interphase and Mitosis. The Journal of Cell Biology. 138, 1193-1206 (1997). [PubMed: 9298976]

49. Lu L, Ladinsky MS, Kirchhausen T, Cisternal Organization of the Endoplasmic Reticulum during Mitosis. Mol. Biol. Cell 20, 3471-3480 (2009). [PubMed: 19494040]

50. Smith JJ, Aitchison JD, Peroxisomes take shape. Nature Reviews Molecular Cell Biology. 14, 803817 (2013). [PubMed: 24263361]

51. Grabenbauer M, Sätzler K, Baumgart E, Fahimi HD, Three-dimensional ultrastructural analysis of peroxisomes in HepG2 cells. Cell Biochem Biophys. 32, 37-49 (2000). [PubMed: 11330069]

52. Veenhuis M, Bonga SEW, Cytochemical localization of catalase and several hydrogen peroxideproducing oxidases in the nucleoids and matrix of rat liver peroxisomes. Histochem J 11, 561-572 (1979). [PubMed: 511592]

53. Angermüller S, Fahimi HD, Selective cytochemical localization of peroxidase, cytochrome oxidase and catalase in rat liver with 3,3' -diaminobenzidine. Histochemistry. 71, 33-44 (1981). [PubMed: 6262282]

54. Titze B, Genoud C, Volume scanning electron microscopy for imaging biological ultrastructure. Biology of the Cell. 108, 307-323 (2016). [PubMed: 27432264]

55. Vidavsky N, Akiva A, Kaplan-Ashiri I, Rechav K, Addadi L, Weiner S, Schertel A, Cryo-FIB-SEM serial milling and block face imaging: Large volume structural analysis of biological tissues preserved close to their native state. Journal of Structural Biology. 196, 487-495 (2016). [PubMed: 27693309]

56. Lizana L, Bauer B, Orwar O, Controlling the rates of biochemical reactions and signaling networks by shape and volume changes. PNAS 105, 4099-4104 (2008). [PubMed: 18337513]

57. Valm AM, Cohen S, Legant WR, Melunis J, Hershberg U, Wait E, Cohen AR, Davidson MW, Betzig E, Lippincott-Schwartz J, Applying systems-level spectral imaging and analysis to reveal the organelle interactome. Nature. 546, 162-167 (2017). [PubMed: 28538724]

58. Alberts B, Ed., Molecular biology of the cell (Garland Science, New York, 4th ed., 2002).

59. Klumperman J, Raposo G, The Complex Ultrastructure of the Endolysosomal System. Cold Spring Harb Perspect Biol 6, a016857 (2014). [PubMed: 24851870]

60. Goldenring JR, Recycling endosomes. Current Opinion in Cell Biology. 35, 117-122 (2015). [PubMed: 26022676]

61. Fermie J, Liv N, ten Brink C, van Donselaar EG, Müller WH, Schieber NL, Schwab Y, Gerritsen HC, Klumperman J, Single organelle dynamics linked to 3D structure by correlative live-cell imaging and 3D electron microscopy. Traffic. 19, 354-369 (2018). [PubMed: 29451726]

62. McGough IJ, Cullen PJ, Recent Advances in Retromer Biology. Traffic. 12, 963-971 (2011). [PubMed: 21463457] 
63. Lecuit T, Adhesion remodeling underlying tissue morphogenesis. Trends in Cell Biology. 15, 3442 (2005). [PubMed: 15653076]

64. McMillen P, Holley SA, Integration of cell-cell and cell-ECM adhesion in vertebrate morphogenesis. Current Opinion in Cell Biology. 36, 48-53 (2015). [PubMed: 26189063]

65. Kanchanawong P, Shtengel G, Pasapera AM, Ramko EB, Davidson MW, Hess HF, Waterman CM, Nanoscale architecture of integrin-based cell adhesions. Nature. 468, 580-584 (2010). [PubMed: 21107430]

66. Bertocchi C, Wang Y, Ravasio A, Hara Y, Wu Y, Sailov T, Baird MA, Davidson MW, Zaidel-Bar R, Toyama Y, Ladoux B, Mege R-M, Kanchanawong P, Nanoscale architecture of cadherin-based cell adhesions. Nature Cell Biology. 19, 28-37 (2017). [PubMed: 27992406]

67. Solecki DJ, Sticky situations: recent advances in control of cell adhesion during neuronal migration. Current Opinion in Neurobiology. 22, 791-798 (2012). [PubMed: 22560352]

68. Gao W-Q, Heintz N, Hatten ME, Cerebellar granule cell neurogenesis is regulated by cell-cell interactions in vitro. Neuron 6, 705-715 (1991). [PubMed: 2025426]

69. Famulski JK, Trivedi N, Howell D, Yang Y, Tong Y, Gilbertson R, Solecki DJ, Siah Regulation of Pard3A Controls Neuronal Cell Adhesion During Germinal Zone Exit. Science. 330, 1834-1838 (2010). [PubMed: 21109632]

70. Chédotal A, Should I stay or should I go? Becoming a granule cell. Trends in Neurosciences. 33, 163-172 (2010). [PubMed: 20138673]

71. de la Torre-Ubieta L, Bonni A, Transcriptional Regulation of Neuronal Polarity and Morphogenesis in the Mammalian Brain. Neuron 72, 22-40 (2011). [PubMed: 21982366]

72. Arrate MP, Rodriguez JM, Tran TM, Brock TA, Cunningham SA, Cloning of Human Junctional Adhesion Molecule 3 (JAM3) and Its Identification as the JAM2 Counter-receptor. J. Biol. Chem 276, 45826-45832 (2001). [PubMed: 11590146]

73. Grimm JB, English BP, Chen J, Slaughter JP, Zhang Z, Revyakin A, Patel R, Macklin JJ, Normanno D, Singer RH, Lionnet T, Lavis LD, A general method to improve fluorophores for live-cell and single-molecule microscopy. Nat Meth 12, 244-250 (2015).

74. Trivedi N, Stabley DR, Cain B, Howell D, Laumonnerie C, Ramahi JS, Temirov J, Kerekes RA, Gordon-Weeks PR, Solecki DJ, Drebrin-mediated microtubule-actomyosin coupling steers cerebellar granule neuron nucleokinesis and migration pathway selection. Nature Communications. 8, 14484 (2017).

75. Simson R, Wallraff E, Faix J, Niewöhner J, Gerisch G, Sackmann E, Membrane Bending Modulus and Adhesion Energy of Wild-Type and Mutant Cells of Dictyostelium Lacking Talin or Cortexillins. Biophysical Journal. 74, 514-522 (1998). [PubMed: 9449351]

76. Lam Hui K, Wang C, Grooman B, Wayt J, Upadhyaya A, Membrane Dynamics Correlate with Formation of Signaling Clusters during Cell Spreading. Biophysical Journal. 102, 1524-1533 (2012). [PubMed: 22500752]

77. Stabley D, Retterer S, Marshall S, Salaita K, Manipulating the lateral diffusion of surface-anchored EGF demonstrates that receptor clustering modulates phosphorylation levels. Integrative Biology. 5, 659 (2013). [PubMed: 23416883]

78. Kuhar SG, Feng L, Vidan S, Ross ME, Hatten ME, Heintz N, Changing patterns of gene expression define four stages of cerebellar granule neuron differentiation. Development. 117, 97104 (1993). [PubMed: 8223263]

79. Frank CL, Liu F, Wijayatunge R, Song L, Biegler MT, Yang MG, Vockley CM, Safi A, Gersbach CA, Crawford GE, West AE, 0896-6273, in press, doi:Neuron.

80. Zhu X, Girardo D, Govek E-E, John K, Mellén M, Tamayo P, Mesirov JP, Hatten ME, Role of Tet1/3 Genes and Chromatin Remodeling Genes in Cerebellar Circuit Formation. Neuron 89, 100112 (2016). [PubMed: 26711116]

81. Chen B-C, Legant WR, Wang K, Shao L, Milkie DE, Davidson MW, Janetopoulos C, Wu XS, Hammer JA, Liu Z, English BP, Mimori-Kiyosue Y, Romero DP, Ritter AT, Lippincott-Schwartz J, Fritz-Laylin L, Mullins RD, Mitchell DM, Bembenek JN, Reymann A-C, Böhme R, Grill SW, Wang JT, Seydoux G, Tulu US, Kiehart DP, Betzig E, Lattice light-sheet microscopy: Imaging molecules to embryos at high spatiotemporal resolution. Science. 346, 1257998 (2014). [PubMed: 25342811] 
82. Ben-Arie N, Bellen HJ, Armstrong DL, McCall AE, Gordadze PR, Guo Q, Matzuk MM, Zoghbi HY, Math1 is essential for genesis of cerebellar granule neurons. Nature. 390, 169 (1997). [PubMed: 9367153]

83. Rose MF, Ren J, Ahmad KA, Chao H-T, Klisch TJ, Flora A, Greer JJ, Zoghbi HY, Math1 Is Essential for the Development of Hindbrain Neurons Critical for Perinatal Breathing. Neuron 64, 341-354 (2009). [PubMed: 19914183]

84. Fanti L, Pimpinelli S, HP1: a functionally multifaceted protein. Current Opinion in Genetics \& Development. 18, 169-174 (2008). [PubMed: 18329871]

85. Elsaesser SJ, Goldberg AD, Allis CD, New functions for an old variant: no substitute for histone H3.3. Current Opinion in Genetics \& Development. 20, 110-117 (2010). [PubMed: 20153629]

86. Sommer C, Straehle C, Köthe U, Hamprecht FA, in 2011 IEEE International Symposium on Biomedical Imaging: From Nano to Macro (2011), pp. 230-233.

87. Delachat AM-F, Guidotti N, Bachmann AL, Meireles-Filho ACA, Pick H, Lechner CC, Deluz C, Deplancke B, Suter DM, Fierz B, Engineered Multivalent Sensors to Detect Coexisting Histone Modifications in Living Stem Cells. Cell Chemical Biology. 25, 51-56.e6 (2018). [PubMed: 29174541]

88. Wong LH, Ren H, Williams E, McGhie J, Ahn S, Sim M, Tam A, Earle E, Anderson MA, Mann J, Choo KHA, Histone H3.3 incorporation provides a unique and functionally essential telomeric chromatin in embryonic stem cells. Genome Res 19, 404-414 (2009). [PubMed: 19196724]

89. Voon HPJ, Wong LH, New players in heterochromatin silencing: histone variant $\mathrm{H} 3.3$ and the ATRX/DAXX chaperone. Nucleic Acids Res 44, 1496-1501 (2016). [PubMed: 26773061]

90. Kukulski W, Schorb M, Kaksonen M, Briggs JAG, Plasma Membrane Reshaping during Endocytosis Is Revealed by Time-Resolved Electron Tomography. Cell. 150, 508-520 (2012). [PubMed: 22863005]

91. Shao L, Isaac B, Uzawa S, Agard DA, Sedat JW, Gustafsson MGL, I5S: Wide-Field Light Microscopy with 100-nm-Scale Resolution in Three Dimensions. Biophysical Journal. 94, 49714983 (2008). [PubMed: 18326649]

92. Shtengel G, Galbraith JA, Galbraith CG, Lippincott-Schwartz J, Gillette JM, Manley S, Sougrat R, Waterman CM, Kanchanawong P, Davidson MW, Fetter RD, Hess HF, Interferometric fluorescent super-resolution microscopy resolves 3D cellular ultrastructure. PNAS 106, 3125-3130 (2009). [PubMed: 19202073]

93. The cryo-SR/FIB-SEM instrument, as described here, will be available at Janelia's Advanced Imaging Center: www.aicjanelia.com.

94. Lepault J, Bigot D, Studer D, Erk I, Freezing of aqueous specimens: an X-ray diffraction study. Journal of Microscopy. 187, 158-166 (1997).

95. Gustafsson MGL, Shao L, Carlton PM, Wang CJR, Golubovskaya IN, Cande WZ, Agard DA, Sedat JW, Three-Dimensional Resolution Doubling in Wide-Field Fluorescence Microscopy by Structured Illumination. Biophysical Journal. 94, 4957-4970 (2008). [PubMed: 18326650]

96. Lowe DG, Distinctive Image Features from Scale-Invariant Keypoints. International Journal of Computer Vision. 60, 91-110 (2004).

97. Betzig E, Patterson GH, Sougrat R, Lindwasser OW, Olenych S, Bonifacino JS, Davidson MW, Lippincott-Schwartz J, Hess HF, Imaging Intracellular Fluorescent Proteins at Nanometer Resolution. Science. 313, 1642-1645 (2006). [PubMed: 16902090]

98. Kopek BG, Shtengel G, Xu CS, Clayton DA, Hess HF, Correlative 3D superresolution fluorescence and electron microscopy reveal the relationship of mitochondrial nucleoids to membranes. Proceedings of the National Academy of Sciences. 109, 6136-6141 (2012).

99. Kopek BG, Shtengel G, Grimm JB, Clayton DA, Hess HF, Correlative Photoactivated Localization and Scanning Electron Microscopy. PLoS ONE. 8, e77209 (2013). [PubMed: 24204771]

100. Perkovic M, Kunz M, Endesfelder U, Bunse S, Wigge C, Yu Z, Hodirnau V-V, Scheffer MP, Seybert A, Malkusch S, Schuman EM, Heilemann M, Frangakis AS, Correlative Light-and Electron Microscopy with chemical tags. Journal of Structural Biology. 186, 205-213 (2014). [PubMed: 24698954] 
101. Watanabe S, Punge A, Hollopeter G, Willig KI, Hobson RJ, Davis MW, Hell SW, Jorgensen EM, Protein localization in electron micrographs using fluorescence nanoscopy. Nat Meth 8, 80-84 (2011).

102. Sochacki KA, Shtengel G, van Engelenburg SB, Hess HF, Taraska JW, Correlative superresolution fluorescence and metal-replica transmission electron microscopy. Nature Methods. 11, 305-308 (2014). [PubMed: 24464288]

103. Löschberger A, Franke C, Krohne G, van de Linde S, Sauer M, Correlative super-resolution fluorescence and electron microscopy of the nuclear pore complex with molecular resolution. $\mathrm{J}$ Cell Sci 127, 4351-4355 (2014). [PubMed: 25146397]

104. Van Engelenburg SB, Shtengel G, Sengupta P, Waki K, Jarnik M, Ablan SD, Freed EO, Hess HF, Lippincott-Schwartz J, Distribution of ESCRT machinery at HIV assembly sites reveals virus scaffolding of ESCRT subunits. Science. 343, 653-656 (2014). [PubMed: 24436186]

105. Vassilopoulos S, Gibaud S, Jimenez A, Caillol G, Leterrier C, "Ultrastructure of the axonal periodic scaffold reveals a braid-like organization of actin rings" (preprint, Cell Biology, 2019), , doi:10.1101/636217.

106. Sartori N, Richter K, Dubochet J, Vitrification depth can be increased more than 10-fold by highpressure freezing. Journal of Microscopy. 172, 55-61 (1993).

107. Richter T, Biel SS, Sattler M, Wenck H, Wittern K-P, Wiesendanger R, Wepf R, Pros and cons: cryo-electron microscopic evaluation of block faces versus cryo-sections from frozen-hydrated skin specimens prepared by different techniques. Journal of Microscopy. 225, 201-207 (2007). [PubMed: 17359255]

108. Li D, Shao L, Chen B-C, Zhang X, Zhang M, Moses B, Milkie DE, Beach JR, Hammer JA, Pasham M, Kirchhausen T, Baird MA, Davidson MW, Xu P, Betzig E, Extended-resolution structured illumination imaging of endocytic and cytoskeletal dynamics. Science. 349, aab3500 (2015). [PubMed: 26315442]

109. Westley MS, Baratta GA, Baragiola RA, Density and index of refraction of water ice films vapor deposited at low temperatures. The Journal of Chemical Physics. 108, 3321-3326 (1998).

110. Török P, Varga P, Laczik Z, Booker GR, Electromagnetic diffraction of light focused through a planar interface between materials of mismatched refractive indices: an integral representation. J. Opt. Soc. Am. A 12, 325 (1995).

111. Egner A, Hell SW, in Handbook Of Biological Confocal Microscopy, Pawley JB, Ed. (Springer US, Boston, MA, 2006; http://link.springer.com/10.1007/978-0-387-45524-2_20), pp. 404-413.

112. Campello RJGB, Moulavi D, Sander J, in Advances in Knowledge Discovery and Data Mining, Pei J, Tseng VS, Cao L, Motoda H, Xu G, Eds. (Springer Berlin Heidelberg, 2013), Lecture Notes in Computer Science, pp. 160-172.

113. Jones E, Oliphant T, Peterson P, SciPy: Open Source Scientific Tools for Python. scipy.org, (available at https://www.scipy.org/).

114. Zack GW, Rogers WE, Latt SA, Automatic measurement of sister chromatid exchange frequency. J Histochem Cytochem 25, 741-753 (1977). [PubMed: 70454]

115. Legant WR, Shao L, Grimm JB, Brown TA, Milkie DE, Avants BB, Lavis LD, Betzig E, High density three-dimensional localization microscopy across large volumes. Nat Methods. 13, 359365 (2016). [PubMed: 26950745]

116. Gatz DF, Smith L, The standard error of a weighted mean concentration-I. Bootstrapping vs other methods. Atmospheric Environment. 29, 1185-1193 (1995).

117. Variance. Wikipedia (2019), (available at https://en.wikipedia.org/w/index.php? title=Variance $\&$ oldid=907622507).

118. Kopek BG, Paez-Segala MG, Shtengel G, Sochacki KA, Sun MG, Wang Y, Xu CS, van Engelenburg SB, Taraska JW, Looger LL, Hess HF, Diverse protocols for correlative superresolution fluorescence imaging and electron microscopy of chemically fixed samples. Nat Protoc 12, 916-946 (2017). [PubMed: 28384138]

119. Buser C, Fleischer F, Mertens T, Michel D, Schmidt V, Walther P, Quantitative investigation of murine cytomegalovirus nucleocapsid interaction. Journal of Microscopy. 228, 78-87 (2007). [PubMed: 17910700] 
120. Blob detection. Wikipedia (2019), (available at https://en.wikipedia.org/w/index.php? title=Blob_detection\&oldid=895846151).

121. Li CH, Tam PKS, An iterative algorithm for minimum cross entropy thresholding. Pattern Recognition Letters. 19, 771-776 (1998).

122. van der Walt S, Schönberger JL, Nunez-Iglesias J, Boulogne F, Warner JD, Yager N, Gouillart E, Yu T, scikit-image: image processing in Python. PeerJ 2, e453 (2014). [PubMed: 25024921]

123. Pedregosa F, Varoquaux G, Gramfort A, Michel V, Thirion B, Grisel O, Blondel M, Prettenhofer P, Weiss R, Dubourg V, Vanderplas J, Passos A, Cournapeau D, Brucher M, Perrot M, Duchesnay É, Journal of Machine Learning Research, in press.

124. Hege H-C, Seebass M, Stalling D, Zöckler M, A Generalized Marching Cubes Algorithm Based on Non-Binary Classifications (1997) (available at https://opus4.kobv.de/opus4-zib/frontdoor/ index/index/docId/274).

125. Taubin G, in Proceedings of the Fifth International Conference on Computer Vision (IEEE Computer Society, Washington, DC, USA, 1995; http://dl.acm.org/citation.cfm? id=839277.840029), ICCV '95, pp. 852-.

126. Bruce MA, Butte MJ, Real-time GPU-based 3D Deconvolution. Opt. Express, OE 21, 4766-4773 (2013).

127. Miura Kota, Rueden Curtis, Hiner Mark, Schindelin Johannes, Rietdorf Jens, ImageJ Plugin CorrectBleach V2.0.2 (Zenodo, 2014; https://zenodo.org/record/30769\#.XVXEDuhKguU).

128. Rego EH, Shao L, Practical Structured Illumination Microscopy. Advanced Fluorescence Microscopy, 175-192 (2015).

129. Liu J, Wu X, Zhang H, Pfeifer GP, Lu Q, Dynamics of RNA Polymerase II Pausing and Bivalent Histone H3 Methylation during Neuronal Differentiation in Brain Development. Cell Reports. 20, 1307-1318 (2017). [PubMed: 28793256]

130. Amrichová J, Lukášová E, Kozubek S, Kozubek M, Nuclear and territorial topography of chromosome telomeres in human lymphocytes. Experimental Cell Research. 289, 11-26 (2003). [PubMed: 12941600]

131. Weierich C, Brero A, Stein S, von Hase J, Cremer C, Cremer T, Solovei I, Three-dimensional arrangements of centromeres and telomeres in nuclei of human and murine lymphocytes. Chromosome Res 11, 485-502 (2003). [PubMed: 12971724]

132. Initial sequencing and comparative analysis of the mouse genome. Nature. 420, 520-562 (2002). [PubMed: 12466850]

133. Richter KN, Revelo NH, Seitz KJ, Helm MS, Sarkar D, Saleeb RS, D’Este E, Eberle J, Wagner E, Vogl C, Lazaro DF, Richter F, Coy-Vergara J, Coceano G, Boyden ES, Duncan RR, Hell SW, Lauterbach MA, Lehnart SE, Moser T, Outeiro TF, Rehling P, Schwappach B, Testa I, Zapiec B, Rizzoli SO, Glyoxal as an alternative fixative to formaldehyde in immunostaining and superresolution microscopy. The EMBO Journal. 37, 139-159 (2018). [PubMed: 29146773]

134. Solecki DJ, Trivedi N, Govek E-E, Kerekes RA, Gleason SS, Hatten ME, Myosin II Motors and F-Actin Dynamics Drive the Coordinated Movement of the Centrosome and Soma during CNS Glial-Guided Neuronal Migration. Neuron 63, 63-80 (2009). [PubMed: 19607793]

135. Hatten ME, Neuronal regulation of astroglial morphology and proliferation in vitro. The Journal of Cell Biology. 100, 384-396 (1985). [PubMed: 3881455]

136. IDL Curve Fitting and Function Optimization, (available at https://www.physics.wisc.edu/ $\sim$ craigm/idl/fitting.html).

137. Brown TA, Tkachuk AN, Shtengel G, Kopek BG, Bogenhagen DF, Hess HF, Clayton DA, Superresolution Fluorescence Imaging of Mitochondrial Nucleoids Reveals Their Spatial Range, Limits, and Membrane Interaction. Molecular and Cellular Biology. 31, 4994-5010 (2011). [PubMed: 22006021]

138. Myronenko A, Xubo Song, Point Set Registration: Coherent Point Drift. IEEE Transactions on Pattern Analysis and Machine Intelligence. 32, 2262-2275 (2010). [PubMed: 20975122]

139. Suleiman H, Zhang L, Roth R, Heuser JE, Miner JH, Shaw AS, Dani A, Nanoscale protein architecture of the kidney glomerular basement membrane. Elife 2, e01149 (2013). [PubMed: 24137544] 
140. Al Jord A, Lemaître A-I, Delgehyr N, Faucourt M, Spassky N, Meunier A, Centriole amplification by mother and daughter centrioles differs in multiciliated cells. Nature (2014), doi:10.1038/nature13770.

141. Wojcik M, Hauser M, Li W, Moon S, Xu K, Graphene-enabled electron microscopy and correlated super-resolution microscopy of wet cells. Nature Communications. 6 (2015), doi: $10.1038 /$ ncomms8384.

142. Lambert TJ, FPbase: a community-editable fluorescent protein database. Nature Methods. 16, 277 (2019). [PubMed: 30886412] 

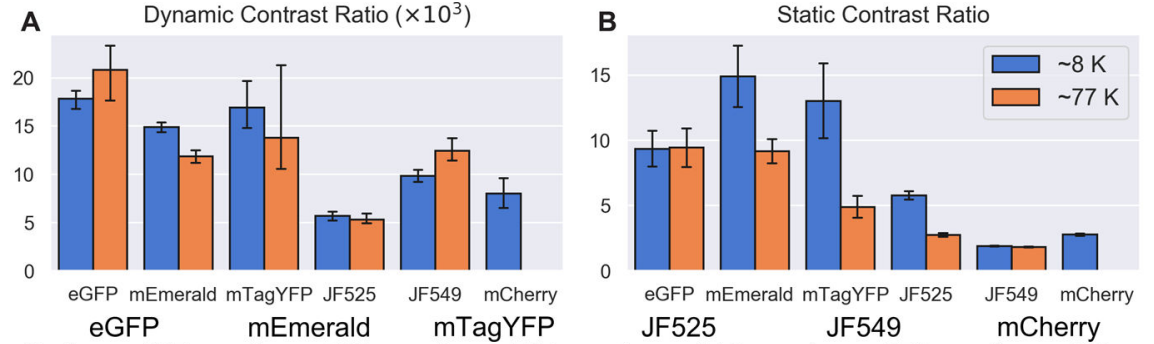

C $\lambda_{\text {em }}=507$
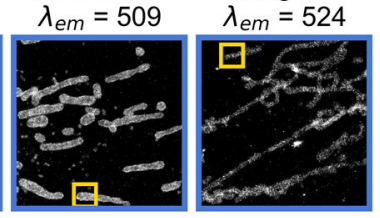

JF525

JF549

mCherry

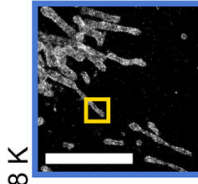

$\lambda_{\mathrm{em}}=548$

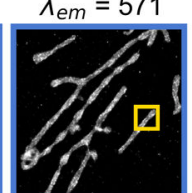

$=610$
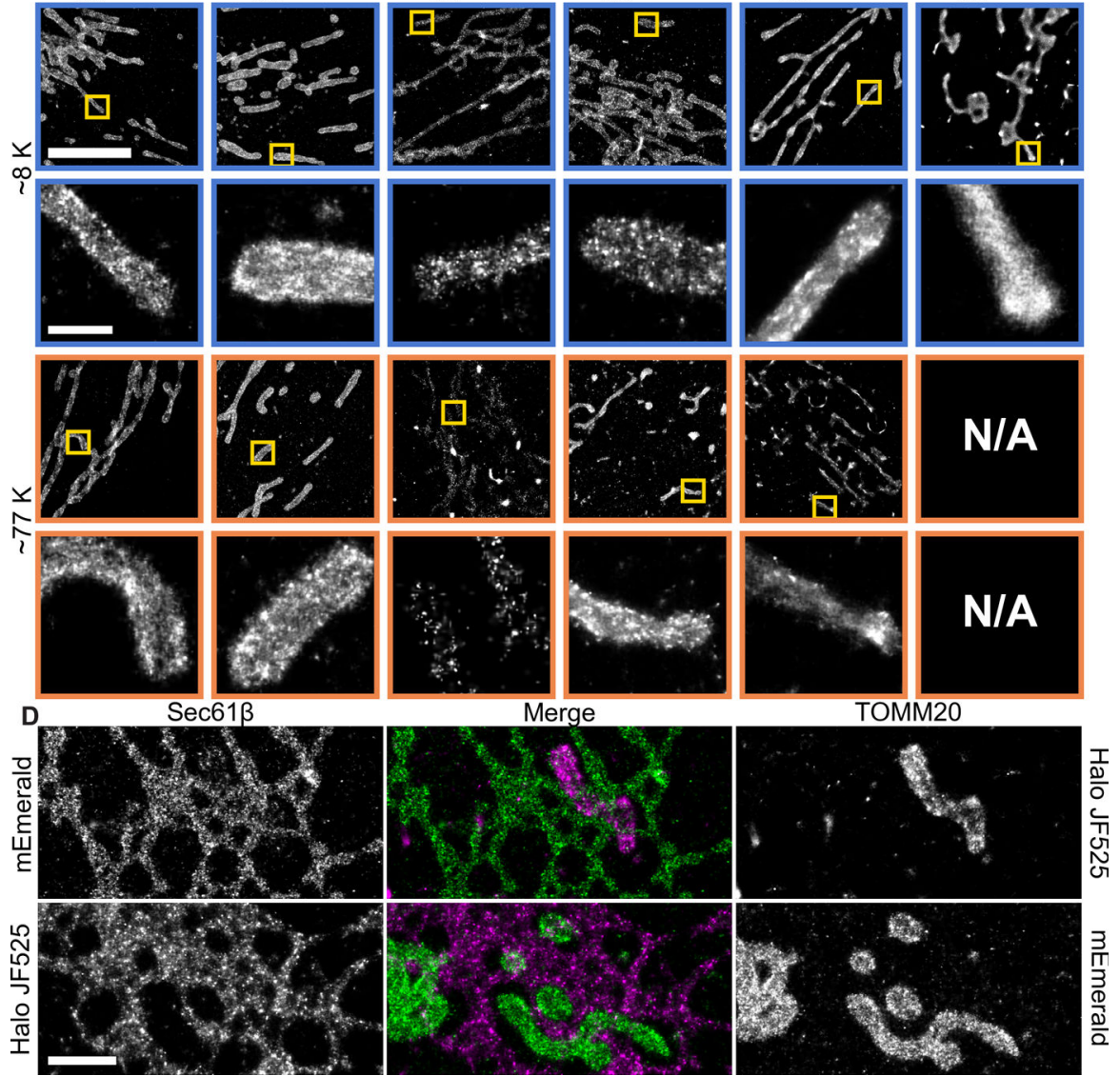

Figure 1. Cryogenic photophysical characterization of fluorophores for single molecule localization microscopy (SMLM)

(A) Dynamic and (B) static contrast ratios for six different fluorophores at $\sim 8 \mathrm{~K}$ (blue) and $\sim 77 \mathrm{~K}$ (orange) ordered by increasing emission wavelength. (C) Corresponding images of mitochondrial outer membrane protein TOMM20 with detailed insets shown below. (D) Comparison of mEmerald and JF525 in cryo-SMLM imaging. Top row: U2OS cell transiently expressing ER membrane marker mEmerald-Sec61 $\beta$ and mitochondrial membrane marker Halo-TOMM20 conjugated to JF525. Bottom row: U2OS cell transiently expressing mEmerald-TOMM20 and Halo/JF525-Sec61 $\beta$. Scale bars: $5 \mu \mathrm{m}$ and $0.5 \mu \mathrm{m}$ in (C), first and second rows; $1 \mu \mathrm{m}$ in (D). 

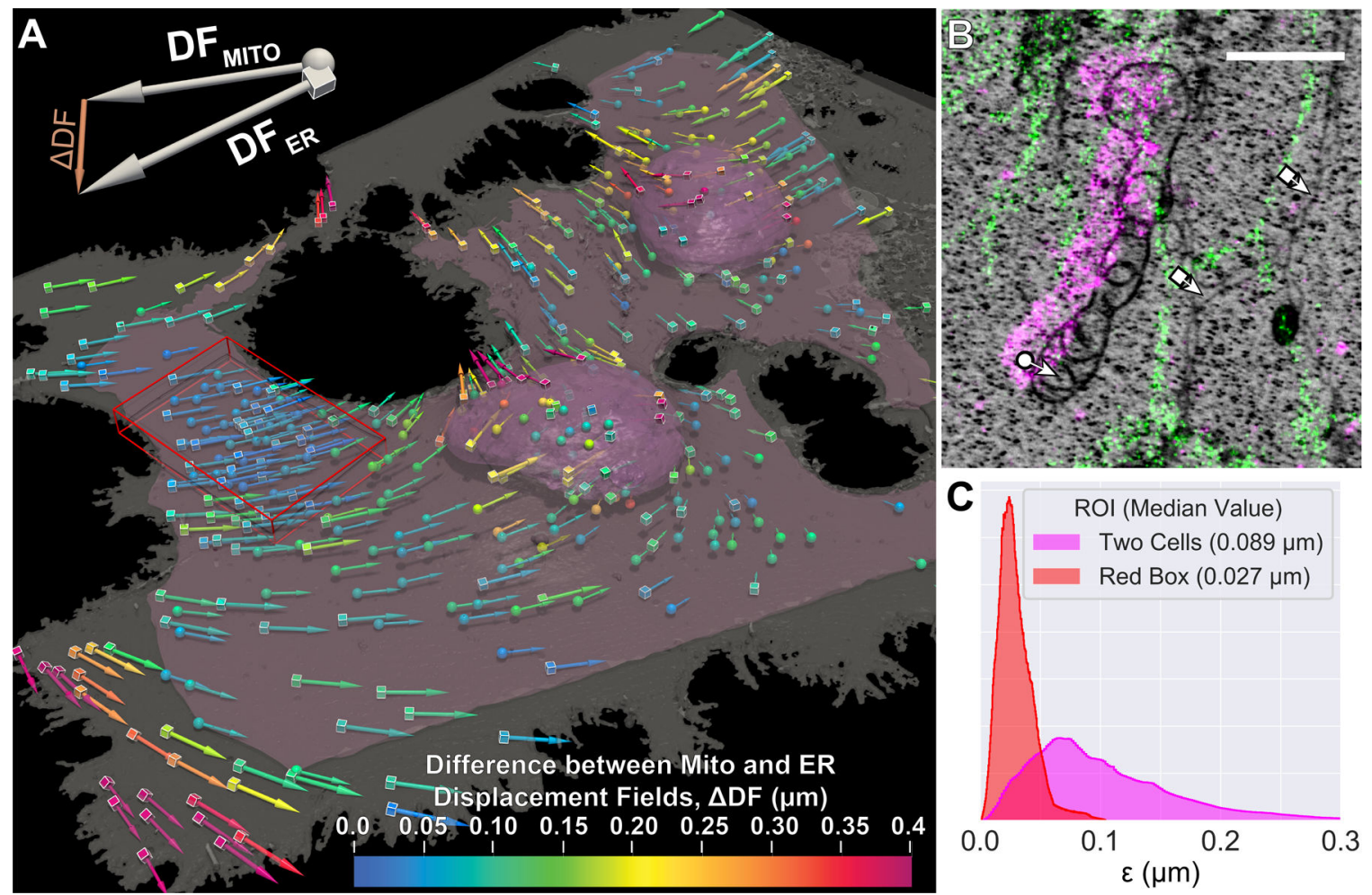

Figure 2. High accuracy correlation of cryo-SMLM and FIB-SEM data using organelle landmarks.

(A) A perspective view of mitochondrial (spheres) and ER (cubes) landmarks used for registration along with the plasma (grey) and nuclear (pink) membranes as determined by FIB-SEM of two COS-7 cells. Arrows point in the direction of and are sized according to the magnitude of the non-linear component of the final displacement field. Arrows are color coded according to the magnitude of the local difference $(\Delta \mathrm{DF})$ between the displacement fields determined by the mitochondrial or the ER landmarks separately. The pink surface indicates the boundaries of the sub-volume containing sufficient landmarks of both types for quantitative comparison of their respective displacement fields. (B) XY orthoslice illustrating landmark selection and determination of the displacement vectors (fig. S14). Scale bar: $1 \mu \mathrm{m}$. (C) Histograms of the correlation accuracy, $\mathcal{\varepsilon}$ (cf. supplementary note 7), for the sub-volume bounded by the pink surface (magenta) and the $61 \mu^{3}$ sub-volume defined by the red box (red), where density of both types of landmarks is higher. 

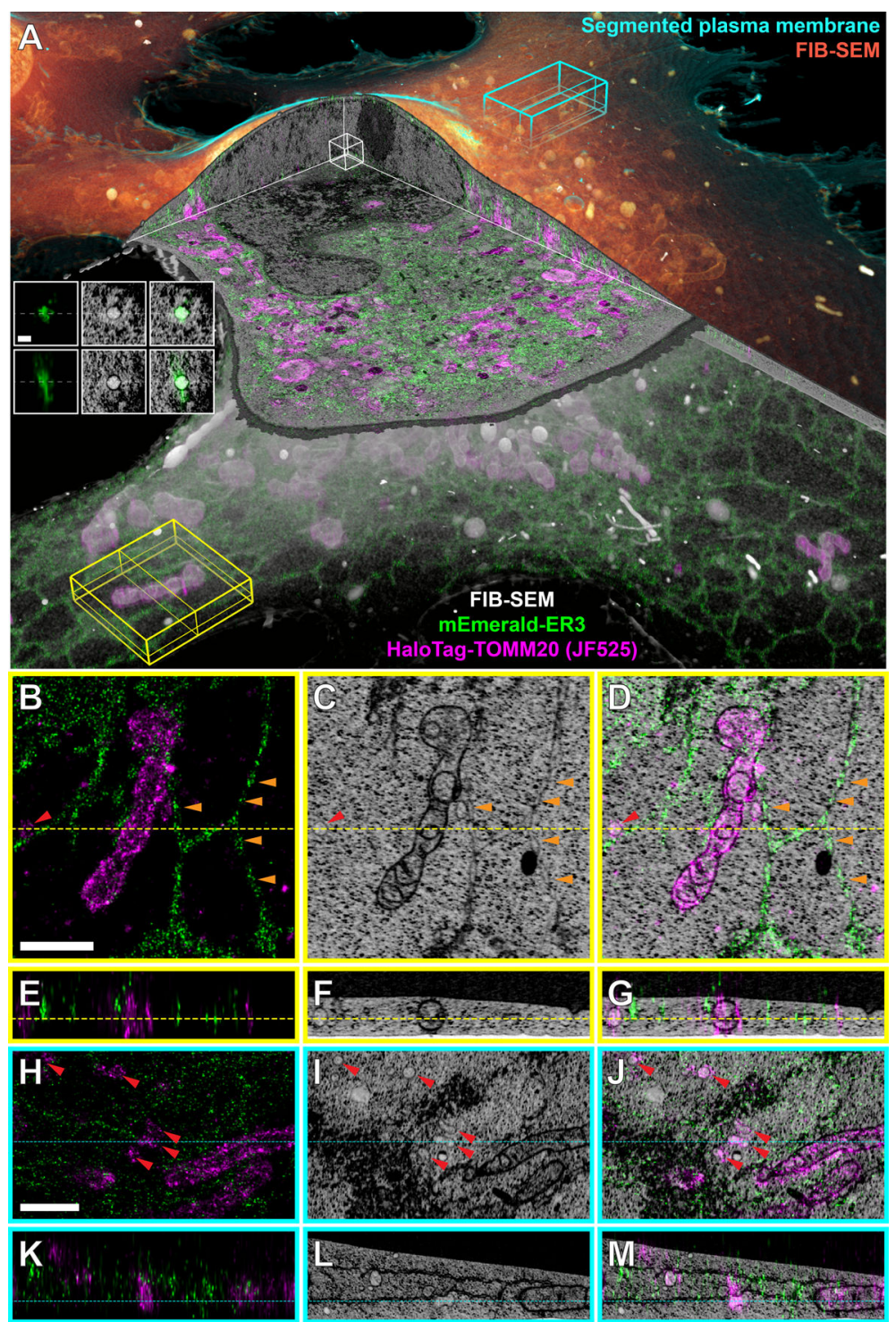

TOMM20-positive vesicles $\triangle \mathrm{ER}$ varicosities

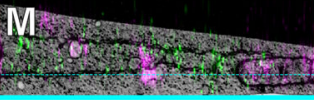

Figure 3. Whole-cell correlative cryogenic single molecule localization and block face electron microscopy.

(A) Perspective overview of a cryo-SMLM and FIB-SEM (orange and grey) data set of a COS-7 cell transiently expressing mEmerald-ER3 (ER lumen marker, green) and Halo/ JF525-TOMM20 (mitochondrial outer membrane marker, magenta) (Movie 2). Cyan, yellow, and white boxes indicate regions with ortho slices shown in panels (B-M) and inset. Inset: SMLM (left column), FIB-SEM (middle column), and correlative (right column) orthoslices in XY (top row) and XZ (bottom row) through an intranuclear ER-positive vesicle. Scale bar: $200 \mathrm{~nm}$. (B, E) SMLM, (C, F) FIB-SEM and (D, G) correlated overlay of orthoslices in XY (B-D) and XZ (E-G) in a lamellipodial region. Scale bar: $1 \mu \mathrm{m}$. (H, K) SMLM, (I, L) FIB-SEM and (J, M) correlated overlay of orthoslices in XY (H-J) and XZ $(\mathrm{K}-\mathrm{M})$ in a thicker region with ER sheets. Scale bar: $1 \mu \mathrm{m}$. Red arrows: TOMM20-positive vesicles; orange arrows: varicosities in the ER. 

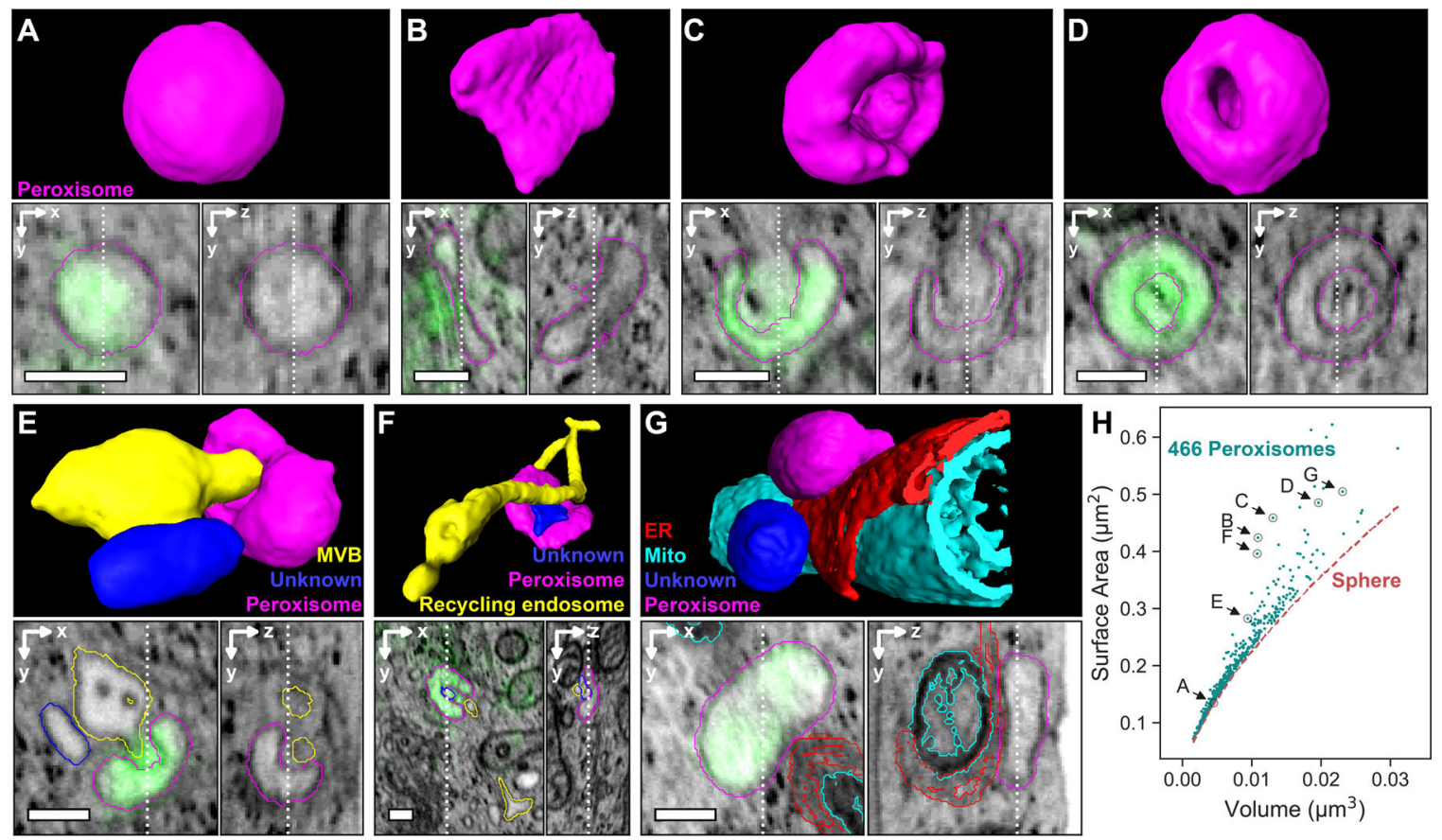

Figure 4. Diversity of peroxisome morphologies and peroxisome-organelle interactions.

(A-D) FIB-SEM segmentations (top) of four peroxisomal targeting signal (SKL) containing peroxisomes (magenta) and corresponding orthoslices (bottom) with cryo-SMLM overlays of SKL (green) from two HeLa cells expressing mEmerald-SKL. (E-G) Three examples of peroxisome/organelle interactions, showing both segmentations (top) and orthoslices (bottom) with overlaid contours of matching colors. Scale bars: $200 \mathrm{~nm}$. (H) Surface-tovolume relationship for 466 peroxisomes (fig. S17), with the specific peroxisomes in (A)$(\mathrm{G})$ indicated, showing the increasing deviation from spherical shape with increasing volume. See also Movie 3. 

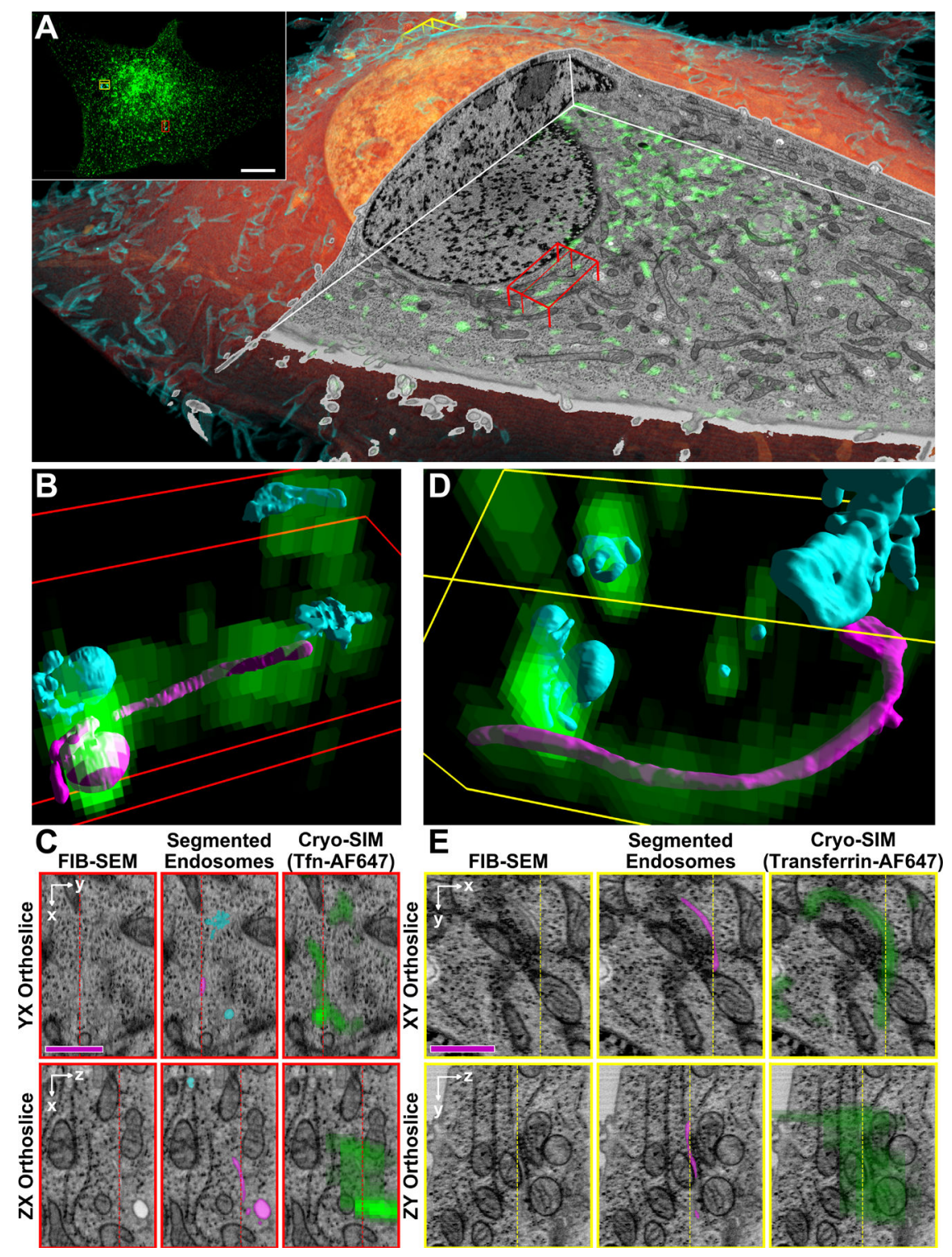

Figure 5. Cryo-SIM/FIB-SEM accurately identifies endosomal compartments and reveals their diverse morphologies at the nanoscale.

(A) Volume rendered FIB-SEM overview (interior, orange; plasma membrane, cyan) of a SUM159 cell, with cutaway correlative cryo-SIM showing endolysosomal compartments containing AF647-conjugated transferrin (green). (B) Segmented Tfn-AF647 containing compartments (colored surfaces) with superimposed 3D cryo-SIM data (green voxels) in the $13 \mu \mathrm{m}^{3}$ subvolume denoted by the red box in (A). (C) XY (top) and ZY (bottom) orthoslices of the same region in (B) showing the FIB-SEM (left) overlaid with segmentations of transferrin labeled compartments (middle) and cryo-SIM of Tfn-AF647 (right). (D, E) Same as (B) and (C) for the $19.5 \mu \mathrm{m}^{3}$ subvolume denoted by the yellow box in (A). Scale bars, (A, inset) $10 \mu \mathrm{m},(\mathrm{C}, \mathrm{E}) 1 \mu \mathrm{m}$. 

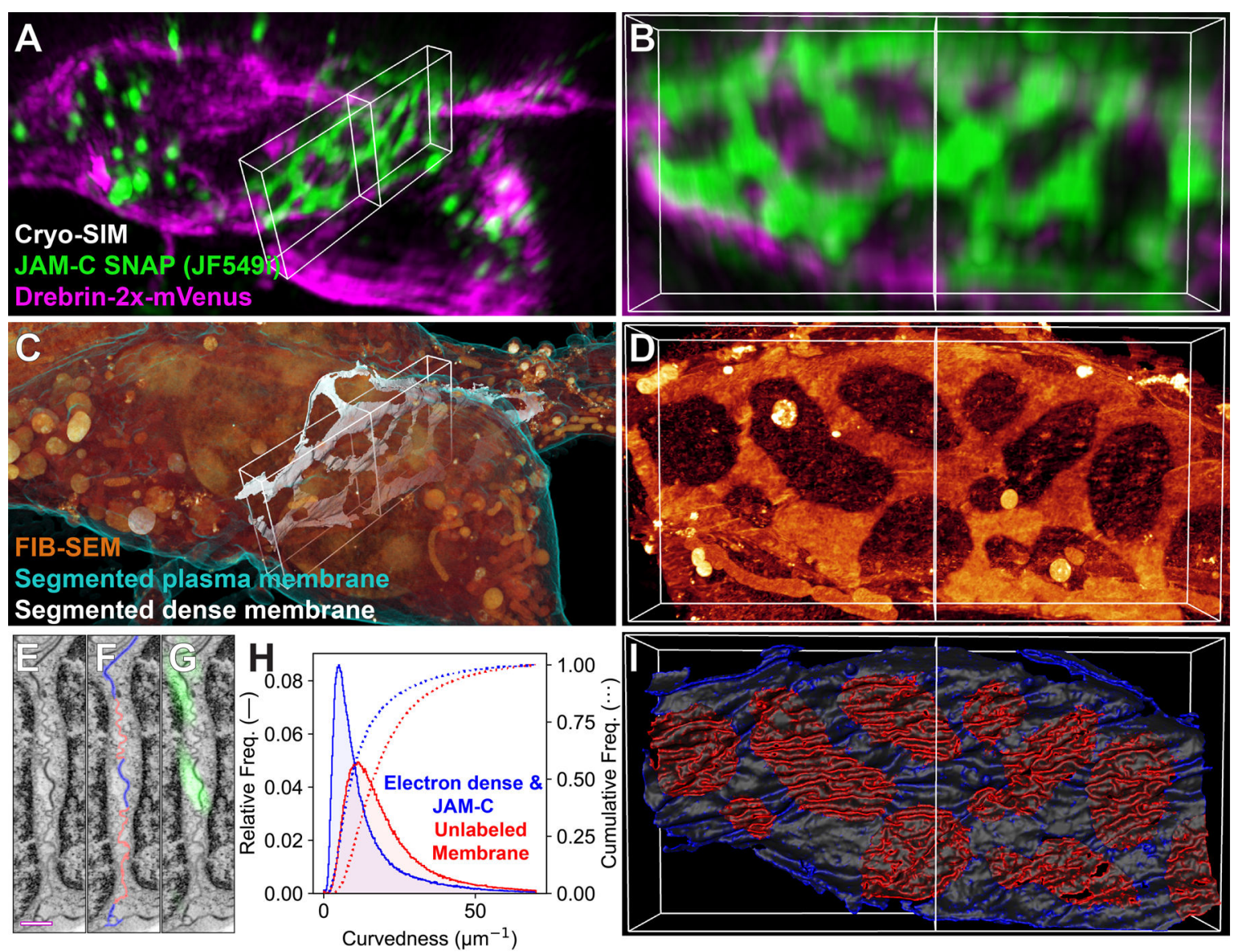

Figure 6. Membrane proteins correlate to membrane ultrastructure at cell-cell adhesions. (A) Cryo-SIM volume of cultured mouse cerebellar granule neurons transiently expressing JF549i/SNAP-JAM-C (green) and 2x-mVenus-Drebrin (magenta). (B) MIP through an $\sim 3$ $\mu \mathrm{m}$ thick slab (white box in (A)) centered on the contact zone between two cell bodies. (C) FIB-SEM volume of the same region in (A), with plasma membrane (cyan), intracellular content (orange), and segmented electron dense regions of the contact zone (white). (D) FIB-SEM MIP through the same region in (B), after masking the nuclei. (E) Single FIBSEM slice through the contact zone at the central vertical line in (D). (F) same as (E), with more (blue) and less (red) electron dense membranes traced; (G) same as (E) overlaid with the JAM-C signal. Scale bar: $500 \mathrm{~nm}$. (H) Histograms of the curvedness (supplemental note 9) for the high (blue) and low (red) electron density membrane regions. (I) Partial segmentation of the cells' membranes in the contact zone, color coded according to curvedness, with brighter colors indicating larger values. Note the high correlation between JAM-C (B), electron density (D), and membrane curvedness (I) (Movie 5). 

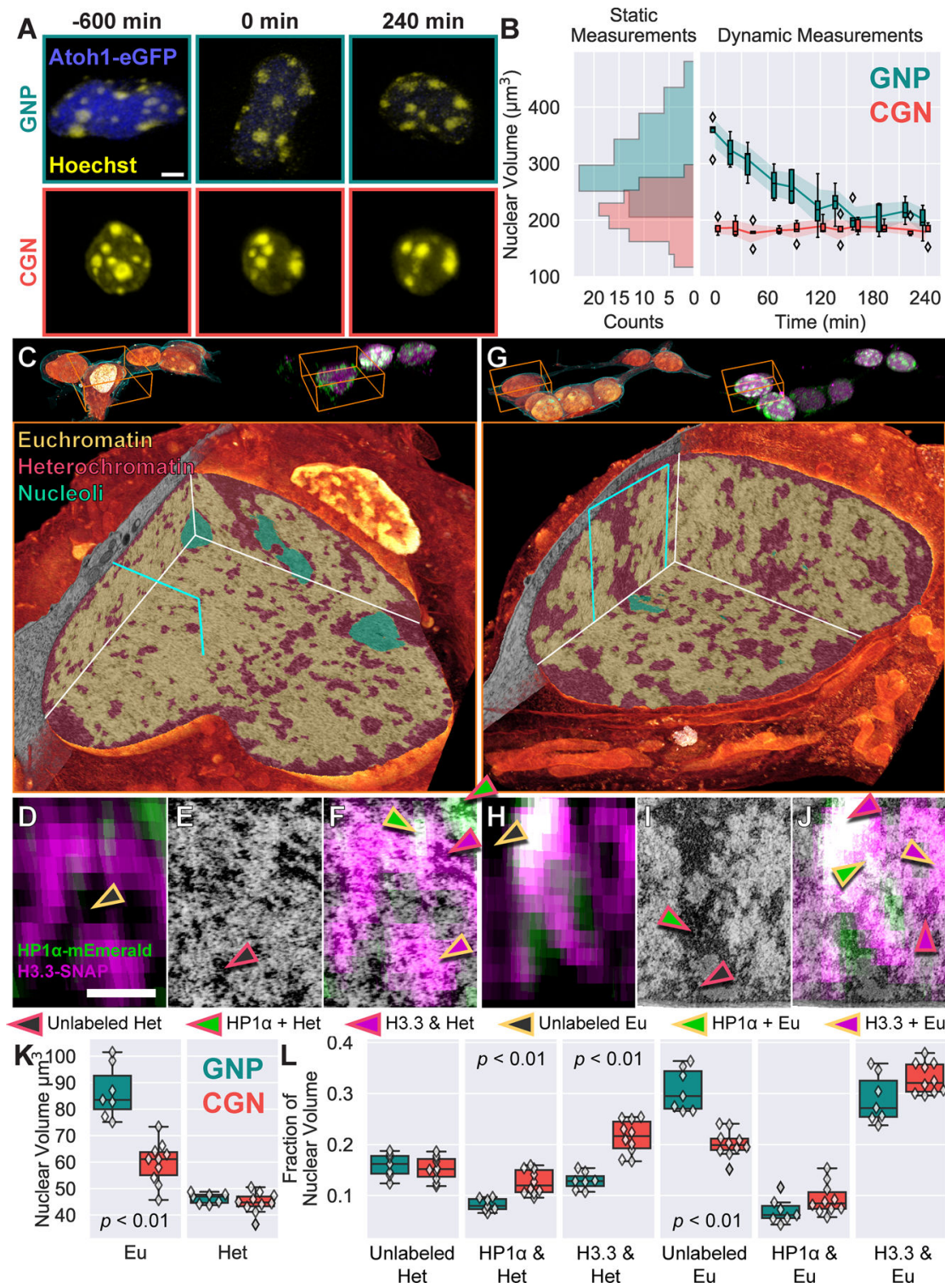

Figure 7. Cryo-SIM/FIB-SEM reveals nuclear rearrangements associated with cerebellar granule neuron progenitor (GNP) differentiation.

(A) Live-cell lattice light sheet time-lapse images showing an EGFP-Atoh1 positive GNP (top row) condensing its nuclear size to that of a CGN while the size of an EGFP-Atoh1 negative CGN nucleus (bottom row) remains constant. Scale bar: $3 \mu \mathrm{m}$. (B) Quantification of GNP and CGN nuclear volume for both static (histograms at left, 85 CGNs and 71 GNPs) and time lapse imaging (box plots at right, 5 GNPs and 5 CGNs), showing that, on average, GNPs are $40 \%$ larger than CGNs and condense their nuclei to the size of CGNs in approximately 2 hours. (C) Top: FIB-SEM (left) and SIM (right) volume renderings of a group of GNPs. Bottom: One such GNP nucleus (orange boxes at top), with cutaway, showing color-coded chromatin territories (heterochromatin, euchromatin or nucleoli) as identified on the basis of the EM data alone. (D-F) HP1a (green) or H3.3 (magenta) CryoSIM, FIB-SEM and correlative XZ ortho slices of the plane bordered in cyan in (C). Arrowheads indicate different types of labeled chromatin domains, see legend. Scalebar, 1 
$\mu \mathrm{m}$. (G-J) Same as (C-F) but for a representative CGN nucleus (Movie 6). (K) Quantification of EM segmented and (L) cryo-SIM defined chromatin domains and their correlation for 7 GNP and 9 CGN nuclei. 
Movie 1. Raw single molecule frames over time since initial illumination illustrating dark state conversion efficiency and background as functions of temperature and emission wavelength. High pressure frozen U2OS cells expressing fluorescent protein or dye labeled TOMM20 to mark the outer mitochondrial membrane, as seen at ten different intervals over 3.5 hours of illumination. Bright continuous emitters are fluorescent bead fiducial markers. As seen, all six emitters asymptote to better single molecule contrast at $\sim 8 \mathrm{~K}$ than $77 \mathrm{~K}$, yielding more accurate single molecule localization (Fig. 1A-C, fig. S9). 
Movie 2. Correlative cryogenic 3D super-resolution and block face electron microscopy of whole vitreously frozen cells.

Two COS-7 cells expressing markers for the endoplasmic reticulum (mEmerald-ER3, green) and mitochondria (Halo/JF535-TOMM20, magenta), shown in relation to orthoslice (grayscale) or volume rendered (plasma membrane, cyan; intracellular volume, orange) FIBSEM data. An ER3-positive intranuclear vesicle and several cytosolic TOMM20-positive vesicles identified by correlation are also highlighted (Fig. 3, fig. S15, S16). 
Movie 3. Structural diversity of peroxisomes and their inter-organelle contacts.

Peroxisomes from a HeLa cell expressing mEmerald-SKL. Part 1 shows orthoslices of the FIB-SEM and cryo-SMLM data followed by segmentations of SKL labeled peroxisomes.

Part 2 shows the same but with segmentations of other organelles in contact with SKL labeled peroxisomes (Fig. 4). 
Movie 4. Cryo-SIM/FIB-SEM reveals the morphological heterogeneity of the endolysosomal system.

A correlative data set of a SUM-159 cell after endosomal uptake of Alexa Fluor-conjugated transferrin. Part 1: 3D cryo-SIM data (green), correlative orthoslices, and correlative volume render (plasma membrane, cyan; cellular interior, orange). Part 2: $\sim 13 \mu^{3}$ sub-volume showing segmentations of all transferrin containing compartments. Part 3: same, but for a different $\sim 20 \mu^{3}$ sub-volume (Fig. 5). 
Movie 5. Correlative cryo-SIM/FIB-SEM reveals a web-like adhesion pattern between adjacent cerebellar granule neurons.

Part 1: cryo-SIM and FIB-SEM volume renderings of a field of CGNs expressing adhesion proteins JAM-C (green) and drebrin (magenta). Part 2: correlation between electron density at the PM, JAM-C cryo-SIM signal, and PM curvature at the interface between two CGNs (Fig. 6). 


\begin{abstract}
Movie 6. Chromatin compaction during differentiation and identification of novel chromatin subdomains.

Correlative data sets of granule progenitor (GNP, left) and cerebellar granule neurons (CGN, right). Part 1: overall correlation between the FIB-SEM (plasma membrane, cyan; cellular interior orange) and cryo-SIM of the nuclear domain reference proteins HP1a (green) and H3.3 (magenta). Part 2: cutaway views of EM-defined chromatin domains for a GNP nucleus (left) and a CGN nucleus (right). Part 3: orthoslices through the CLEM volumes indicating subdomains defined by overlap between EM-defined nuclear domains and nuclear domain reference proteins. Part 4: 3D surface renderings of CLEM defined nuclear chromatin subdomains for the GNP and CGN nuclei (Fig. 7).
\end{abstract}

